\title{
Measuring the One-Way Speed of Light
}

\author{
Kenneth William Davies ${ }^{1}$ \\ ${ }^{1}$ New Westminster, British Columbia, Canada \\ Correspondence: Kenneth William Davies, New Westminster, British Columbia, Canada. E-mail: \\ ken@imgmaker.com \\ Received: October 18, 2018 \\ Accepted: November 9, 2018 \\ Online Published: November 30, 2018 \\ doi:10.5539/apr.v10n6p45 \\ URL: https://doi.org/10.5539/apr.v10n6p45
}

\begin{abstract}
This paper describes a method for determining the one-way speed of light.

My thesis is that the one-way speed of light is NOT constant in a moving frame of reference, and that the one-way speed of light in any moving frame of reference is anisotropic, in that its one-way measured speed varies depending on the direction of travel of light relative to the direction of travel and velocity of the moving frame of reference.

Using the disclosed method for measuring the one-way speed of light, a method is proposed for how to use this knowledge to synchronize clocks, and how to calculate the absolute velocity and direction of movement of a moving frame of reference through absolute spacetime using the measured one-way speed of light as the only point of reference.
\end{abstract}

Keywords: One-Way Speed of Light, Clock Synchronization, Absolute Space and Time, Lorentz Factor

\section{Introduction}

The most abundant particle in the universe is the photon. A photon is a massless quanta emitted by an electron orbiting an atom. A photon travels as a wave in a straight line through spacetime at the speed of light, and collapses to a point when absorbed by an electron orbiting an atom.

There are three commonly proven facts about the speed limit of light:

1) The speed of light is constant in all frames of reference.

2) Nothing in the universe travels faster than the speed of light.

3) Objects gain relativistic mass as they are accelerated. Trying to accelerate an object to the speed of light is impossible as it requires an infinite amount of energy as the mass goes to infinity.

Two less known facts encapsulated by Einstein in his theory of Special Relativity published in 1905, are that an object compress in length in the direction of travel as the object's velocity approaches the speed of light. Also, that time itself slows down or dilates within an object that is moving at close to the speed of light.

For example, a clock that is flying through space at $50 \%$ the speed of light is compressed in the direction of movement to $86.6 \%$ of its length, and time slows by a factor of 1.155 , so that 100 minutes as measured by the travelling clock is actually 115.5 minutes as measured by a stationary observer.

Gravity also distorts time and space, as described in Einstein's theory of General Relativity published in 1915. Space is compressed in the direction of the gravitational force, and time slows down as the gravitational attraction increases. In the extreme case of a black hole, to an outside observer, an object crossing the event horizon essentially becomes two dimensional, and all time within the object stops.

The principle of relativity states that you can't perceive these time and space differences inside your own frame of reference, but you can perceive them in an object that is moving through your frame of reference. Although a stationary observer sees a space ship flying past at 50\% the speed of light as being compressed in length, and with slow running clocks, inside the space ship, everything appears to be normal. There is no measurable horizontal compression in the direction of travel, and all of physics works as it should, including the measurement of time, distance, and the speed of light. 
Even stranger is the claim that without any additional knowledge, you can't tell which frame of reference is moving, and which frame of reference is stationary. Is the spaceship travelling past you, or are you travelling past the space ship? Everything is relative.

No one can explain why space and time vary based on velocity and gravitational forces.... it just IS.

\subsection{Practical Tests of Relativity:}

The idea that time and space are not constant continues to be tested and proven as new technologies are introduced into the world.

For example, GPS satellites have extremely accurate clocks that have to be adjusted for time slowing down due to their fast orbital speed, but also time is speeding up because the satellites are further outside of the Earth's gravity well. GPS clocks also are adjusted for differences in the one-way speed of light caused by the Sagnac Effect. A linear accelerators that smash atoms together works because of relativistic effects that happen when mass is accelerated to $99.9 \%$ of the speed of light. We have also discovered that the centres of galaxies are anchored by giant black holes whose gravity potential is so immense that not even light itself can escape, and for any physical object that falls into the black hole's event horizon, all time stops.

\subsection{What is Time and Space?}

According to the International System of Units (SI) definitions:

- A second is defined as exactly 9,192,631,770 times the period of the radiation corresponding to the transition between the two hyperfine levels of the ground state of the caesium-133 atom.

- A meter is defined as the length of the path travelled by light in a vacuum during a time interval of $1 / 299,792,458$ of a second.

- $\quad$ The speed of light in a vacuum is defined as $299,792,458$ metres per second.

According to Einstein, the only invariant quantity in all frames of reference is the speed of light. Time and Space are variant. This proposition leads to the following logical contradiction: Since the speed of light is defined as distance travelled through space in a unit of time, it would seem that if space and time change based on relativistic effects within a frame of reference, so too should the measured value for the speed of light.

What is mind boggling about the theory of relativity is that space and time change in the exact amount required so that the measured speed of light 'appears' to be the same in all frames of reference. It's a trick of the universe that as we start going faster through space, or as we are drawn into a gravity well, all the laws of physics continue to operate to our perception as though nothing has changed, even though space is clearly compressing, and time is clearly slowing down - as measured by a person outside the moving frame of reference.

\subsection{Time Dilation}

In a frame of reference that is moving at a measurable fraction of the speed of light, or is situated in a gravity well, time slows down. At the speed of light, or behind the event horizon of a black hole, time ceases to exist. A photon exists in a timeless state.

By saying 'time slows down', what we are saying is that the fundamental frequency, at which a climate controlled caesium-133 atom vibrates, slows down. Chemical reactions slow down. Decay rates of fundamental particles slow down. Quantum interactions slow down. Explosive reactions slow down. The orbital speed of an electron around a nucleus slows down. Every measurable or quantifiable physical property of mater that is repetitive in nature slows down.

Another example used to explain time dilation is as follows: There are two twins, one that stays on Earth, and a second that goes into a space ship that travels at $99.9 \%$ the speed of light in a big loop, and returns back to Earth 22 years later. On the space-ship's return, the twin that stayed on Earth is biologically 22 years older, but the twin in the space ship is biologically only one year older.

\subsection{Length Contraction}

In the same frame of reference where time slows down, the object compresses in length in the direction of travel. Like a fun-house full of mirrors, only outside observers can perceive that inside the object, time and space have changed. For example, in particle accelerators, where protons are accelerated to $99.9999 \%$ the speed of light, the spherical protons gain mass, and behave like a flattened disk when smashing into other objects. 


\subsection{Relativistic Doppler Effect}

Photons travel at the speed of light. If a moving object emits a photon of frequency $\mathbf{f}$ in the forward direction, that photon travels at the speed of light but at a higher frequency $\mathbf{f}+$. If that same moving object emits a photon of frequency $\mathbf{f}$ in the reverse direction, that photon travels at the speed of light, but at a lower frequency $\mathbf{f}$ There is also a Doppler effect on photons that travel out of gravity well: the photons travel out of the well at the speed of light, but at a reduced frequency. As frequency equates to energy, you can also say that photons gain or lose energy depending on the launch velocity from the object the photos were generated from.

Objects that are travelling away from us emit photons that appear to us as red-shifted (lower frequency/lower energy). Objects that are travelling towards us emit photons that are blue shifted (higher frequency/higher energy).

Photons within a spaceship travel forward at a frequency $\mathbf{f}+$, bounce off the forward wall, and return to our eye at a frequency of $\mathbf{f}$-, but because our eye is also moving forward, we perceive the color as frequency $\mathbf{f}$. In this fashion, light reflecting around a room in a moving object appears to us to behave the same way as light reflecting around a room in a non-moving object.

\subsection{Measuring Time and Space is Relative}

It is possible that a synchronized event that occurs at the same time in one frame of reference may appear to occur at a different unsynchronized times in a second moving frame of reference. Even though this happens, you still can't use this knowledge to tell which frame of reference is stationary, and which frame is moving.

\subsection{One-Way Speed of Light}

To date no successful experiment has conclusively measured the one-way speed of light.

In the real world, the two-way speed of light is physically measured using a light source, a single clock, and a mirror. A light beam is sent in the direction of the mirror at the clock time of 00:00, the light beam reflects back from the mirror, and then arrives back at the starting point, where the clock then stops. The stop time of the clock is the speed of light over that measured distance. The longer the distance used to measure the speed, the more accurate the results. In most cases, the light pulse is sent back and forth a number of times between mirrors to increase the distance of travel before being finally measured.

If the mirror is one half a kilometre away, and the light pulse gets sent, bounces off the mirror, and then returns to the source in some fraction of a second ' $t$ ' to stop the clock, the two-way speed of light is defined to be one kilometre per fraction of a second ' $t$ ', which is then normally restated as kilometres per second.

Measuring the speed of light in only one direction is difficult because of clock synchronization problems. You have to synchronized the start and stop clocks. Two side-by-side clocks might start out synchronized, but as you move them away from each other, due to relativistic effects caused by the movement of the second clock, the exact time between the two clocks may not remain the same.

The second issue to overcome measuring the one-way speed of light is you can't use the same trick of bouncing the light pulse back and forth between mirrors to lengthen the measurement time and thus the measurement accuracy. It's a direct line of sight measurement. So your clocks have to be fairly far apart, and super accurate.

Finally, no one believes that the speed of light in one direction is any different than the two-way speed of light. The issue was decided more than 100 years ago, all of science still works, so it's not an issue high up on any-one's to-do list to get excited about.

\subsection{Can Neutrinos travel faster than the speed of light?}

Neutrinos are point like particles that are generated in nuclear environments, like the Sun or in a fission reactor. At any point in time there are billions of low energy neutrinos streaming from the Sun through you and through the Earth. It's actually very difficult to capture a neutrino or know if a neutrino has passed through you because low energy neutrinos don't normally interact with matter.

High energy neutrinos on the other hand rarely make it through the Earth's atmosphere before hitting something, and causing a shower of short lived particles.

One of the scientific questions currently being investigated is at what speed do neutrinos travel? Is it at the speed of light, meaning neutrinos have no mass, or is it at one billionth a percentage less than the speed of light because neutrinos have some infinitesimally small fractional mass?

If you can figure out the speed of a neutrino, then you can calculate the mass, and if you know the mass, then 
you can do all sorts of more detailed calculations with regard to the physical properties of matter that produce neutrinos.

Every now and then someone buried deep in a research laboratory yells out they think they just measured the speed of a neutrino that travelled faster than the speed of light. But, on closer inspection, the results can't be duplicated, and the general consensus is the experiment was in error.

Why I bring this up is that the speed of a neutrino can only be measured in one direction. And we know that neutrinos travel at either the speed of light, or a very close approximation to the speed of light. Speed measurements of neutrinos are going to be plagued with the same clock synchronization issues used to measure the one-way speed of light, making it difficult to independently test and confirm the speed measurement results.

If we can solve the problem of measuring the one-way speed of light, that may lead to better techniques for synchronizing clocks, and measuring the one-way speed of neutrinos. And if we can accurately measure the speed of neutrinos, we might just catch one going faster than the speed of light.

\section{Calculating the One-Way speed of light}

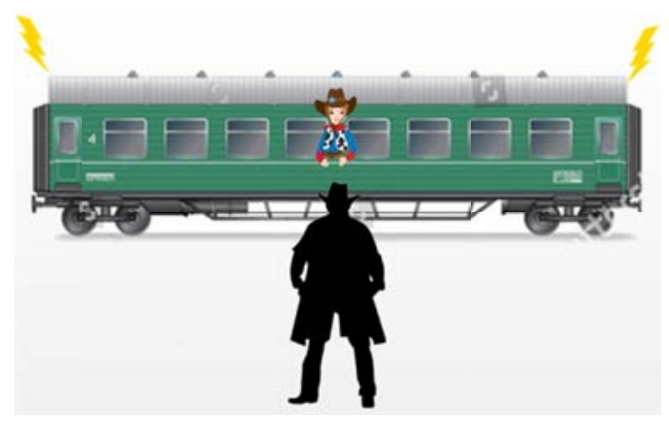

Figure 1.

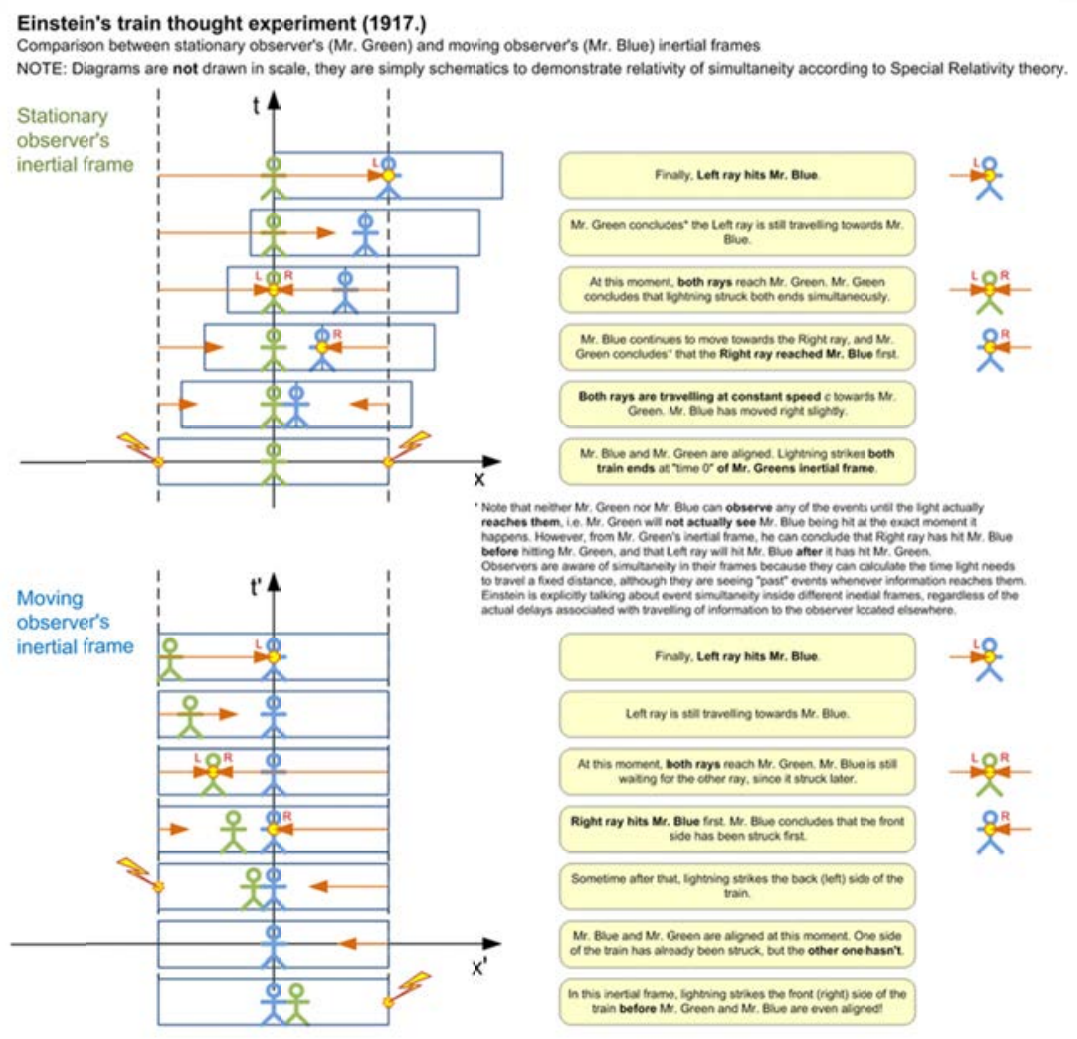

Figure 2.

Source: "Relativity of" (2018) 


\subsection{Relativity of Simultaneity}

Einstein imagined a stationary observer who witnessed two lightning bolts simultaneously striking both ends of a moving train. He concluded that an observer standing on the moving train would see the bolts striking at different times from what the stationary observer saw.

This strange behaviour of light within a moving object leads to all sorts of odd predictions.

Below is a screen capture from a Wikipedia page that shows how each frame of reference views the event.

In my opinion there is a logic flaw in the Moving Observer's Inertial Frame diagram. The corrected scenario is that the lightning strikes both sides at exactly the same time, but that the forward one-way speed of light inside the moving object is different from the reverse one-way speed of light. The difference in speed of light is the reason the moving observer sees the strike from the right before they see the strike from the left.

On the next page is the corrected diagram.

\subsection{A Corrected Moving Inertial Frame Diagram}

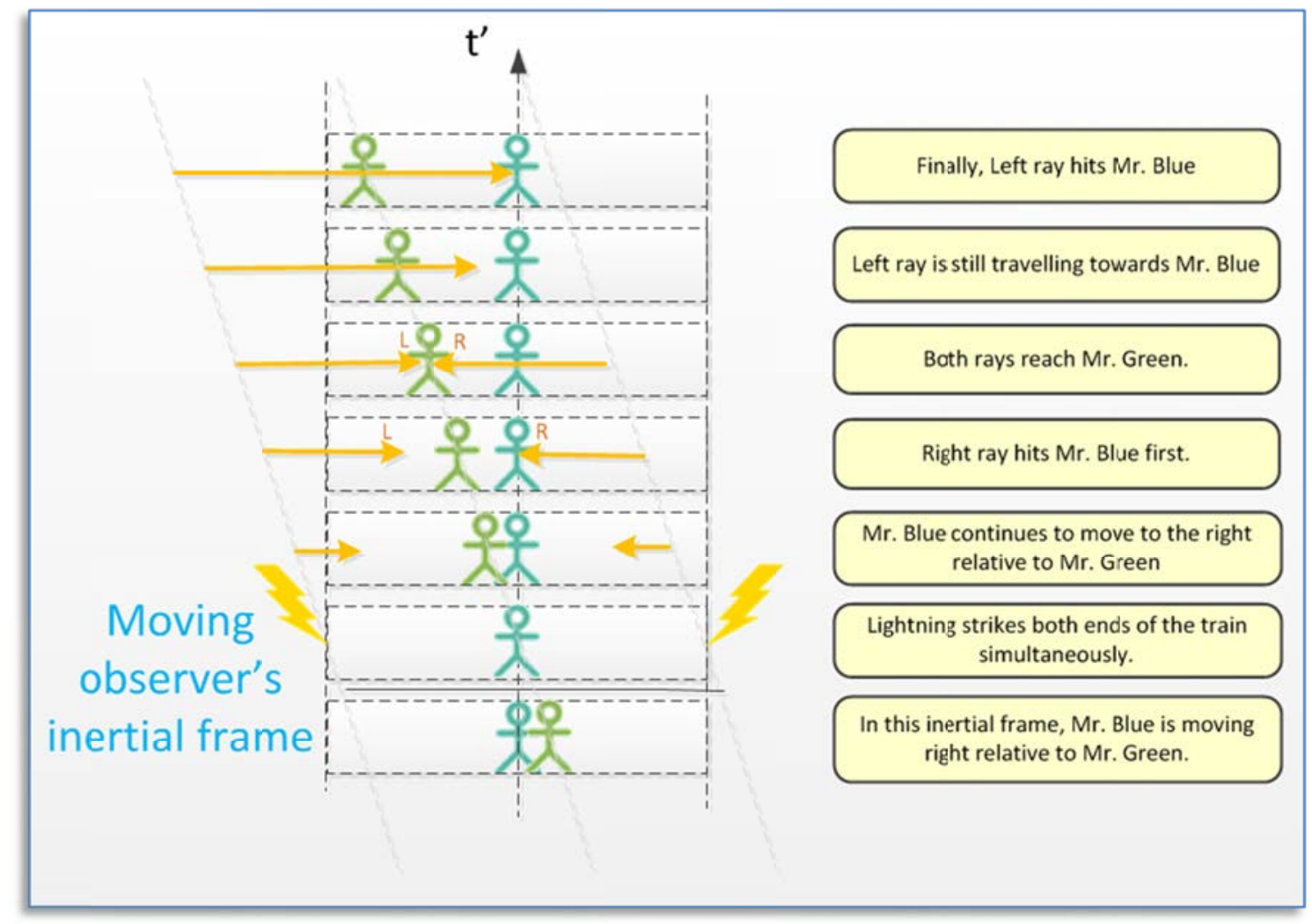

Figure 3.

Note that the light rays travel at the same speed in both directions in the stationary frame of reference, but from the perspective of the person in the moving frame of reference, the Right ray travels faster than the Left ray.

The problem in trying to prove this is that there are no obvious ways to measure the one-way speed of light.

When the Right light ray hits Mr. Blue, it could be argued that because the speed of light is constant, the lightning bolt must have hit the right side before it hit the left side. Since making a direct measurement of the one-way speed of light is impossible, there is no way to argue which of the two moving observers' inertial frame diagrams is correct.

For the rest of this paper, I'm going to break with convention, and argue that the one-way speed of light is measurable, and that the one-way speed of light in a moving frame of reference is not a constant.

\subsection{Light behaviour in a moving object}

If you trace the path of two photons emitted simultaneously, one in each direction, from a light source enclosed in a moving box travelling at 50\% the speed of light, you get the following diagram through time: 


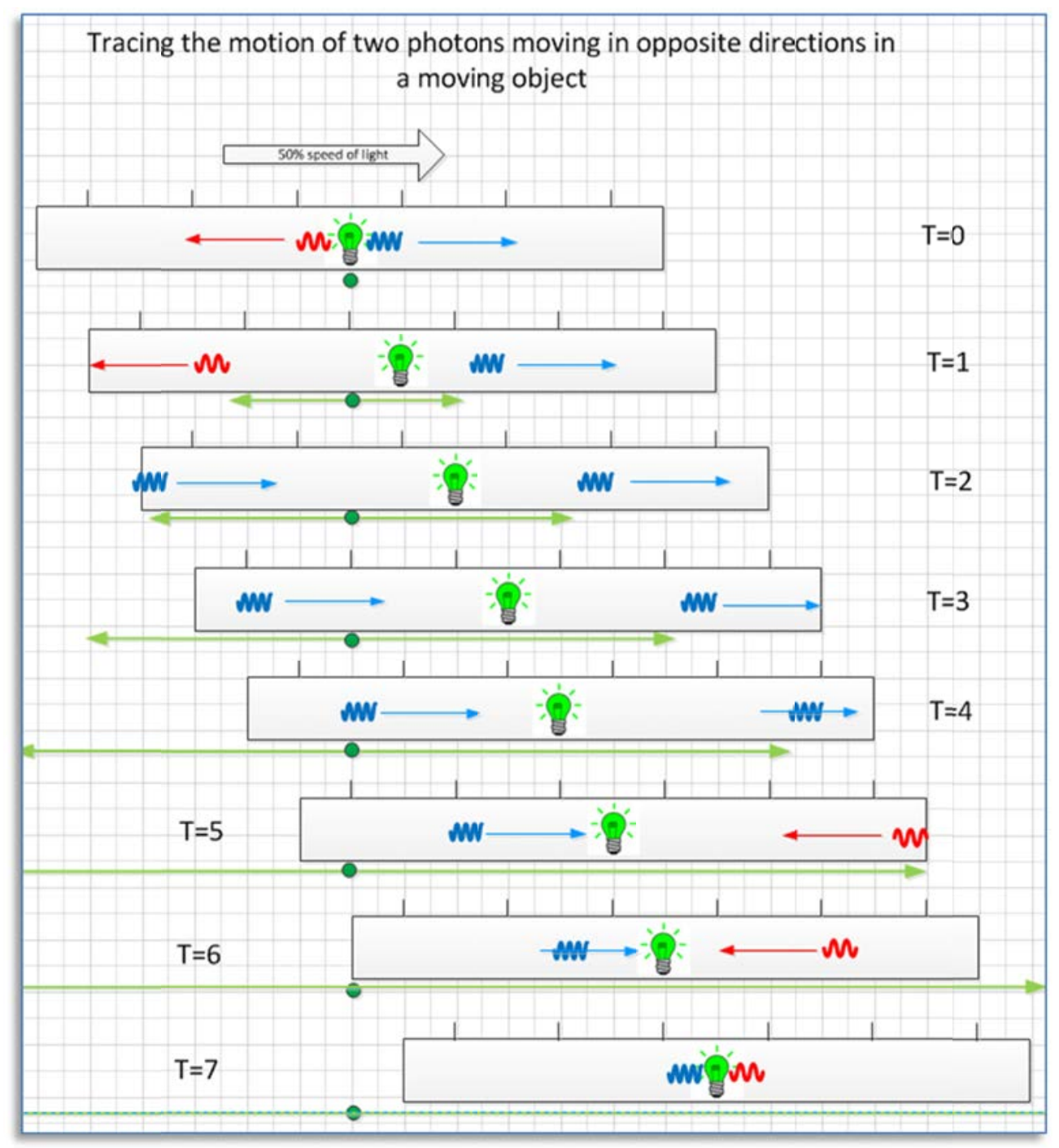

Figure 4.

For comparison, a stationary green light outside of the box emits two photons synchronized to the emitted photons within the moving box. The speed of light plotted inside the moving box is the same as the speed of light plotted outside of the moving box, as the absolute speed of light is constant in all frames of reference.

Total distance travelled in the box is the same for each pulse. The average two-way speed of light is C.

Note that from the perspective of a person standing inside the box, the forward one-way speed of light in the moving frame of reference (blue photon) is considerably slower than the reversed one-way speed of light (red photon). The two-way speed of light remains constant.

\subsection{Calculating the One-Way Speed of a Horse}

Below is an example calculation for the one-way speed of a horse. 


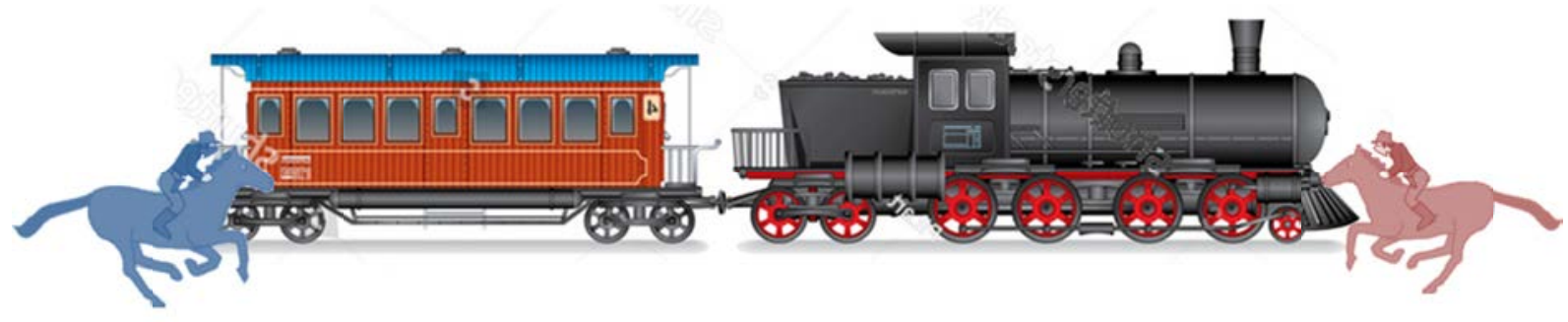

Figure 5 .

Speed of Train: $10 \mathrm{KM} / \mathrm{hr}$

Speed of Blue Horse: $20 \mathrm{KM} / \mathrm{hr}$

Speed of Red Horse: $\quad-20 \mathrm{KM} / \mathrm{hr}$

Length of Train: $1 \mathrm{KM}$

Equation to calculate time to reach other end of the train:

Time $=$ Length of Train / absolute (speed of horse - speed of train)

Red horse travel time: $\quad \mathrm{T}=1 / \mathrm{abs}(-20-10)=0.033 \mathrm{hr}$

Blue horse travel time: $\quad \mathrm{T}=1 / \mathrm{abs}(20-10)=0.100 \mathrm{hr}$

Red horse speed relative to a moving train is $3 \mathrm{x}$ times faster than Blue horse.

The horses run $2 \mathrm{x}$ times faster than the train. In the forward direction, for every two meters the horse travels across the ground, the train moves ahead one meter. The net result is that on the ground the blue horse covers two meters, but from the frame of reference of someone on the train, the blue horse has only advanced one meter.

In the reverse direction, for every two meters the red horse travels on the ground, the train moves back one meter. The net result is that on the ground the red horse covers two meters, but from the frame of reference of someone on the train, the red horse has advanced three meters.

The red horse travels $3 \mathrm{x}$ faster when going from front to back than the blue horse travelling back to front of a moving train.

\subsection{Calculating the One-Way Speed of Light}

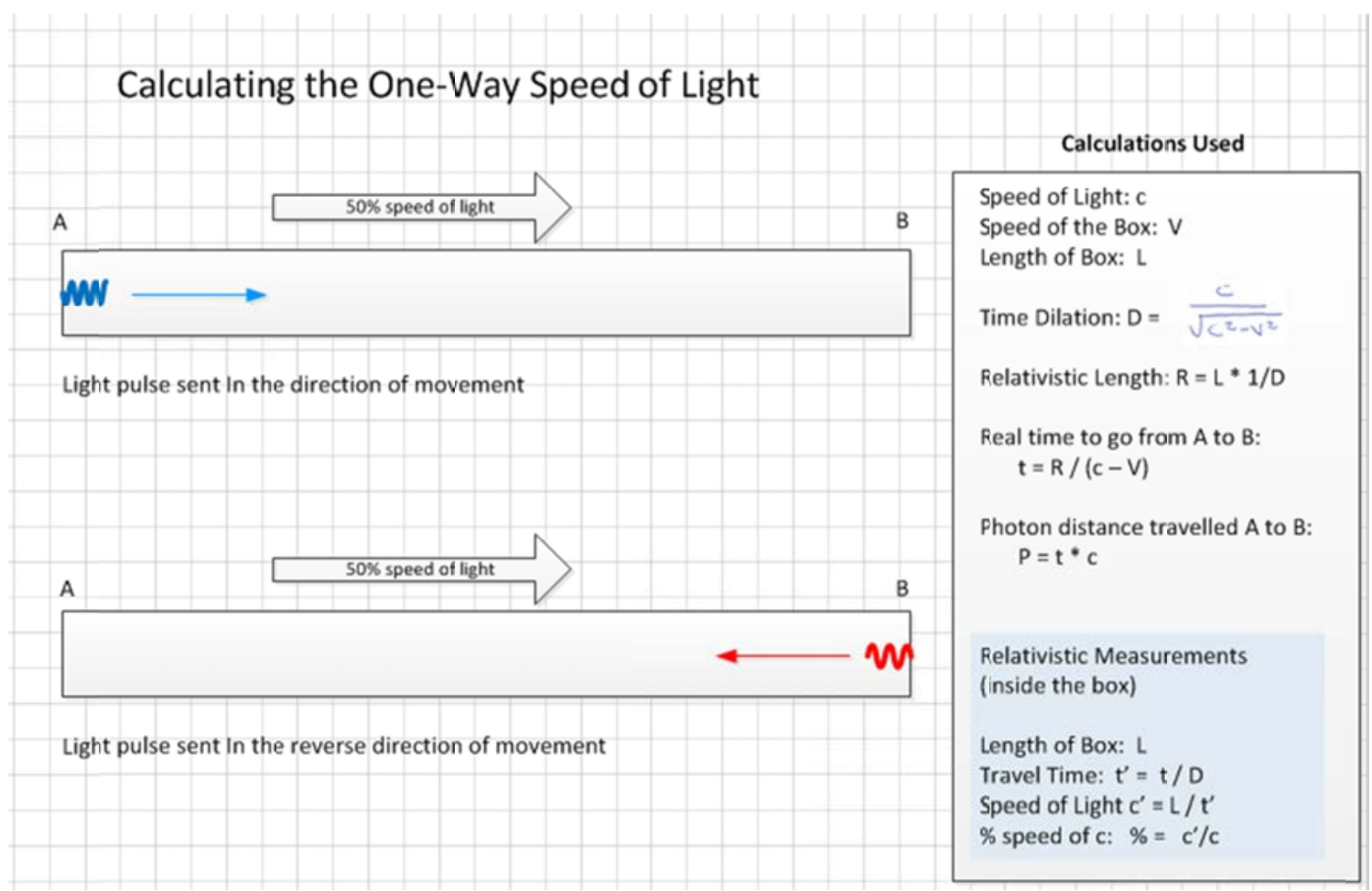




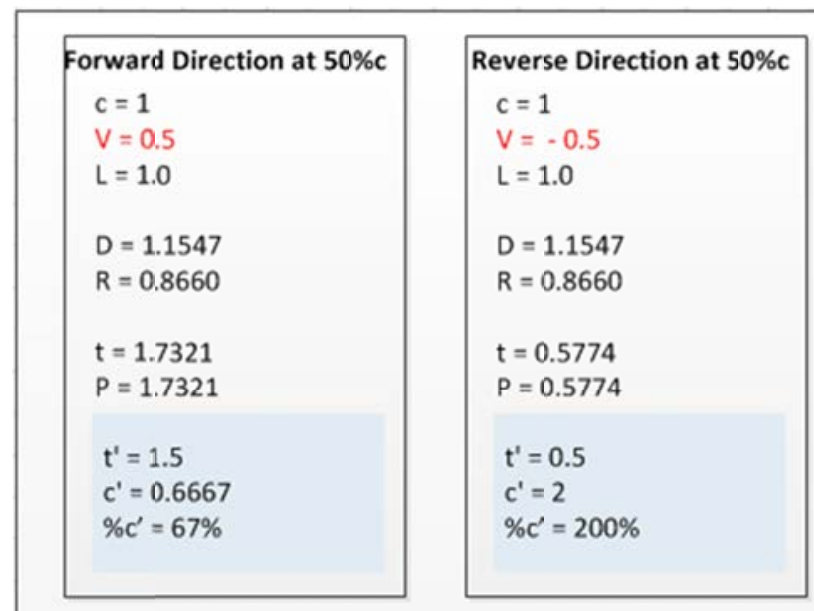

In a box that is one light speed second in length, travelling at $50 \%$ the speed of light, it takes light 1.5 seconds to reach the end in the direction of travel, and 0.5 seconds to return.

The one-way speed of light in the foreword direction is $67 \%$ the speed of light. The one-way speed of light in the reverse direction is $200 \%$ the speed of light.

The reverse direction is $3 \times$ faster than the forward direction.

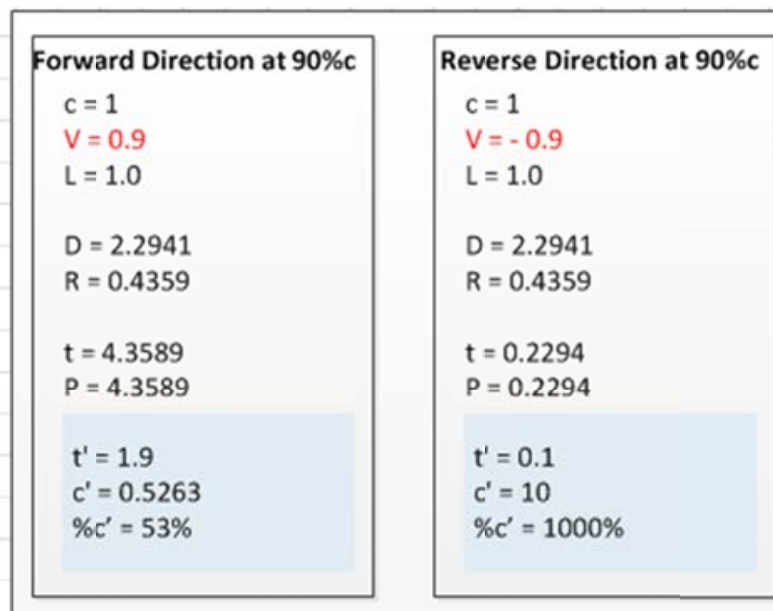

In a box that is one light speed second in length, travelling at $90 \%$ the speed of light, it takes light 1.9 seconds to reach the end in the direction of travel, and 0.1 seconds to return.

The one-way speed of light in the foreword direction is 53\% the speed of light. The one-way speed of light in the reverse direction is $1000 \%$ the speed of light.

The reverse direction is $19 \mathrm{x}$ faster than the forward direction

Figure 6.

\section{A Thought Experiment for Measuring the One-Way Speed of Light}

The problem measuring the speed of light in only one direction is that no one has figured out how to synchronize the clocks. For our first experiment, let's ignore the idea of synchronized clocks. What we are going to use is highly accurate unsynchronized clocks.

For reference, caesium atomic clocks are accurate to 10 to the -13 seconds. A nanosecond (or 10 to the -9 seconds) is the time it takes for light to travel 30 centimetres. 10 to the -13 is the time it takes for light to travel 0.03 millimetres, and is one tenth of a picosecond.

Below is an experiment to time the one-way speed of light, as measured inside a moving frame of reference.

\subsection{The Experimental Set-Up is as Follows:}

Imagine a large disk 100 meters in radius, with one clock and a green laser secured at the centre, pointing to a second clock and a photo detector secured to the outside circumference of the disk. The clocks aren't synchronized, but they are highly accurate time-keepers. 


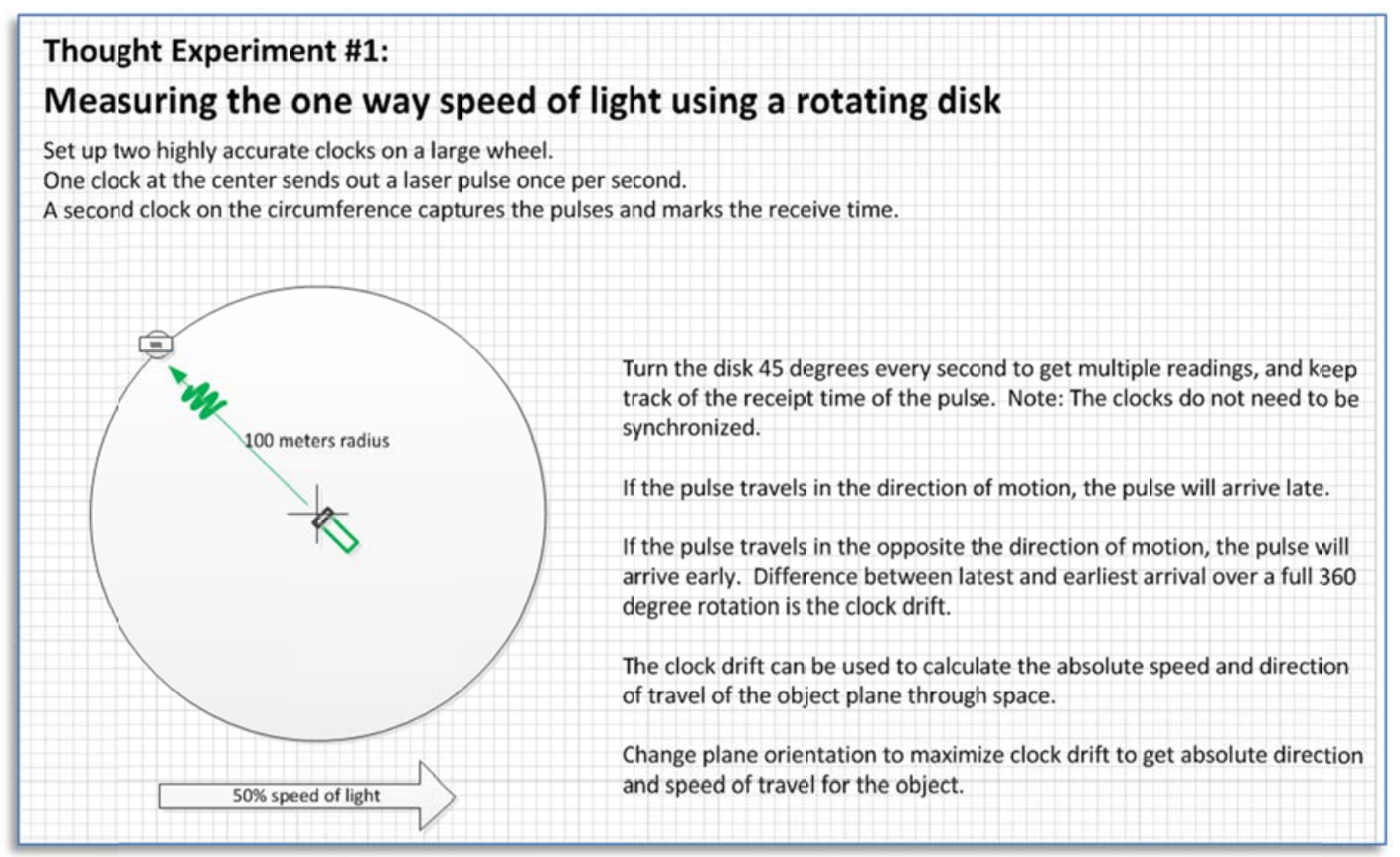

Figure 7.

For simplicity I've drawn the green laser clock in the centre of the disk. I can just as easily fix the green laser clock to the circumference of the disk so as to remove the possibility that one clock is moving at a different speed relative to the other (and will in fact do so in a later iteration of this experiment). I have not accounted for the time dilatation or length contraction issues, as we are conducting this experiment from the frame of reference of the person that is travelling on the disk.

The laser clock is set up to fire a pulse of green laser light once every second, exactly on the second. The clock on the circumference is set up to detect the pulse, and to record the exact time the pulse was received. Since the clocks aren't synchronized, we haven't measured anything to do with light speed just yet. But what we can measure with extreme accuracy is that the received light pulse arrives once a second, at exactly one second after the previous pulse was measured, to an accuracy of 10 to the -13 seconds.

Now, place this disk in space, without the influence of any gravitational field, travelling at $50 \%$ the speed of light. Also, the disk is now rotating very slowly, with a completed 360 degree rotation once every 8 seconds. Assume the rotation stops momentarily when taking each of the 8 measurements. 


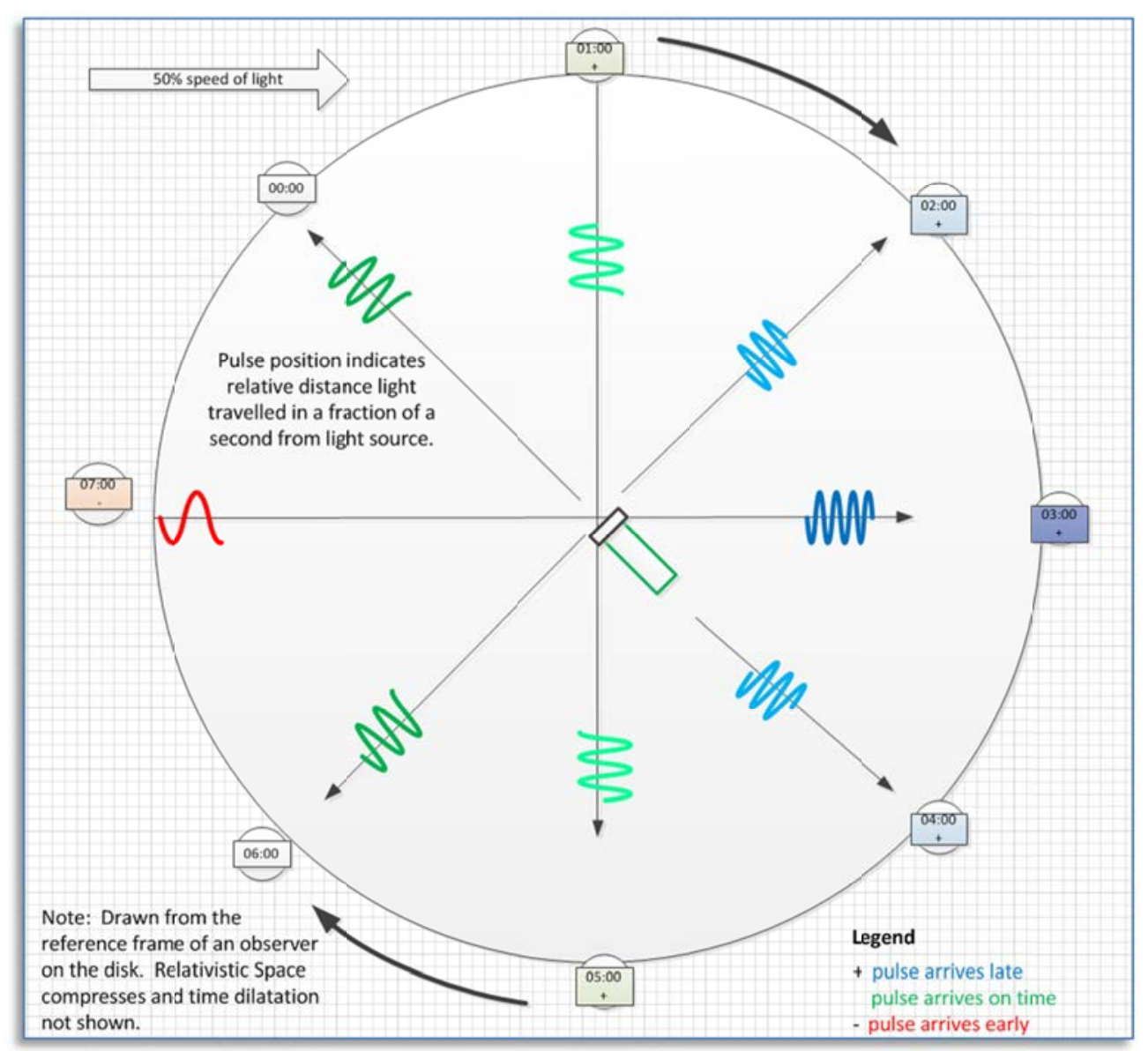

Figure 8 .

Note that I've drawn the green laser light with different frequency values to account for relativistic Doppler Shift. When pointed in a forward direction, the light frequency increases to blue and ultra violet. When pointed in the backwards direction, the light frequency lengthens to red and infra-red. The light frequency shift would not be noticeable to the person on the disk because when the light hits a receptor, the forward speed of the receptor fixed to the disk restores the light frequency back to green.

From the perspective of a person on the disk, light travelling in the forward direction of motion travels slower across the radius of the disk than light travelling in the reverse direction of motion. This is because the disk is travelling at 50\% the speed of light, and in the forward direction light travels two meters for every one meter that the disk travels forward, (for a net gain of one meter), and in the backward direction, light travels two meters backwards for every one meter the disk travels forward, (for a net gain of three meters). The maximum speed ratio difference between the forward and reverse one-way speed of light is 3:1.

If the one-way light pulse is travelling at different speeds across the radius of the disk, and the clock is keeping accurate time as to the exact point in time when the light pulse arrives at the circumference for each of the measured angles through a complete rotation of the disk, the capture time will drift around the one second tick mark.

\subsection{Measuring Clock Drift}

The pulse is being sent once a second from the center of the disk, but is being received slightly before or after the second-tick on the clock positioned on the rotating circumference. Again, the clocks aren't synchronized, but they are keeping track of their own version as to when a second expires. If the pulse arrives early or late on the circumference due to a difference in travel time, it will be recorded as such by the second clock.

The slowest measured pulse time (maximum drift value) indicates the direction of travel of the disk plane through spacetime, and if the two-way speed of light is measured for the radius, then we can infer the two 
one-way speeds of light across the radius from the measured maximal and minimal drift values. The two $\mathrm{max} / \mathrm{min}$ range values for the one-way speed of light are the one-way speed of light minus $1 / 2$ the drift range, and the one-way speed plus $1 / 2$ the drift range.

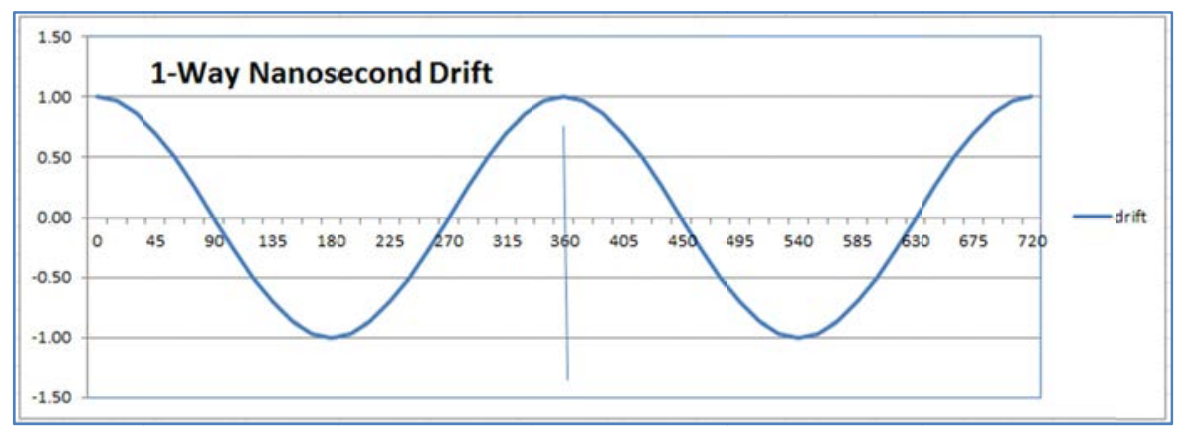

Figure 10.

Drift range of 2 nanoseconds. Fastest one-way light speed occurs at 180 degree rotation.

Direction of travel of the object is at $\mathbf{0}$ and 360 degrees rotation.

Using real-world numbers, and measured in the frame of reference of the moving disk: If the radius of the disk is 100 meters, and light travels at 299792.458 kilometres per second in a vacuum, and the disk is moving at $50 \%$ the speed of light, the two-way time light takes to cross 100 meters is 3.3356E-07 seconds. The fastest one-way time to cross the radius is $1.66782 \mathrm{E}-07$, and the slowest one-way time to cross the radius is $5.00346 \mathrm{E}-07$. The maximal drift is 3.33564E-07 seconds, or 333.6 nanoseconds.

Converted back to Kilometres per second, the two measured max and min times for the speed of light are: $599584.916 \mathrm{KM} / \mathrm{sec}$ or $200 \%$ the two-way speed of light, and $199861.6387 \mathrm{KM} / \mathrm{sec}$ or $66.666667 \%$ the two-way speed of light. The ratio between the fastest and slowest one-way light speed is $3: 1$.

\begin{tabular}{|c|c|c|c|c|}
\hline 4 & A & B & C & D \\
\hline 1 & \multicolumn{2}{|c|}{ calculations in KM per second } & \multirow[b]{2}{*}{ there } & \multirow[b]{2}{*}{ back } \\
\hline 2 & & & & \\
\hline 3 & & speed of light & 299792.458 & 299792.458 \\
\hline 4 & & speed of box & 149896.229 & -149896.229 \\
\hline 5 & & length of box & 0.1 & 0.1 \\
\hline 6 & & time dilation & 1.154700538 & 1.154700538 \\
\hline 7 & & relativisticlength & 0.08660254 & 0.08660254 \\
\hline \multicolumn{5}{|l|}{8} \\
\hline 9 & photon & & & \\
\hline 10 & & travel time & $5.7775 E-07$ & 1.92583E-J7 \\
\hline 11 & & total time: & & 7.70333E-J7 \\
\hline 12 & & distance travelled & 0.173205081 & 0.057735027 \\
\hline 13 & & total distance & & $0.2309401) 8$ \\
\hline \multicolumn{5}{|l|}{14} \\
\hline 15 & \multicolumn{2}{|c|}{ As measured outside the moving box: } & & \\
\hline 16 & & 1 way speed of light & 299792.458 & 299792.458 \\
\hline 17 & & 2 way speed of light & & 299792.458 \\
\hline \multicolumn{5}{|l|}{18} \\
\hline 19 & \multicolumn{4}{|c|}{ As measured inside the moving box: } \\
\hline 20 & \multirow{3}{*}{\multicolumn{2}{|c|}{$\begin{array}{l}\text { length of box } \\
\text { total distance }\end{array}$}} & \multirow[t]{2}{*}{0.1} & C.1 \\
\hline 21 & & & & C.2 \\
\hline 22 & & travel time & \multirow[t]{2}{*}{$5.00346 \mathrm{E}-07^{\prime}$} & $1.66782 \mathrm{E}-37$ \\
\hline 23 & & total time & & $6.67128 \mathrm{E}-37$ \\
\hline 24 & & 1 way speed of light & \multirow{3}{*}{199861.6387} & 599584.916 \\
\hline 25 & & 2 way speed of light & & 299792.458 \\
\hline \multicolumn{4}{|l|}{26} & \\
\hline 27 & & $\% 1$ way speed & \multirow[t]{4}{*}{$66.666667 \%$} & $200.00000 \mathrm{C} \%$ \\
\hline 28 & & $\% 2$ way speed & & $100.00000 c \%$ \\
\hline 29 & & & & \\
\hline 30 & & drift in seconds: & & $3.33564 E-J 7$ \\
\hline
\end{tabular}

Figure 11. 


\subsection{Measuring Clock Drift through different $Z$ spin axis orientations}

The largest positive drift value measured when rotating the disk orientation through a fixed $Z$ spin axis indicates the $\mathrm{X} / \mathrm{Y}$ forward direction of movement through space for that $\mathrm{Z}$ plane. To get the maximal $\mathrm{X} / \mathrm{Y} / \mathrm{Z}$ value of travel, rotate the disk plane through 180 degrees of the $Z$ spin axis, while repeatedly testing to obtain the maximal $\mathrm{X} / \mathrm{Y}$ directional drift value for each $\mathrm{Z}$.

After identifying the maximal $X$ and $Y$ drift value for all values for $Z$, you've now located the true $X / Y / Z$ vector direction of motion through absolute space. You can then use the drift value obtained to reverse calculate the fastest and slowest one-way speeds of light, and then use the ratio of fastest to slowest to reverse lookup the speed of the object through spacetime.

A maximal drift value of 0 through all rotational $Z$ planes indicates a Dead-Stop in spacetime.

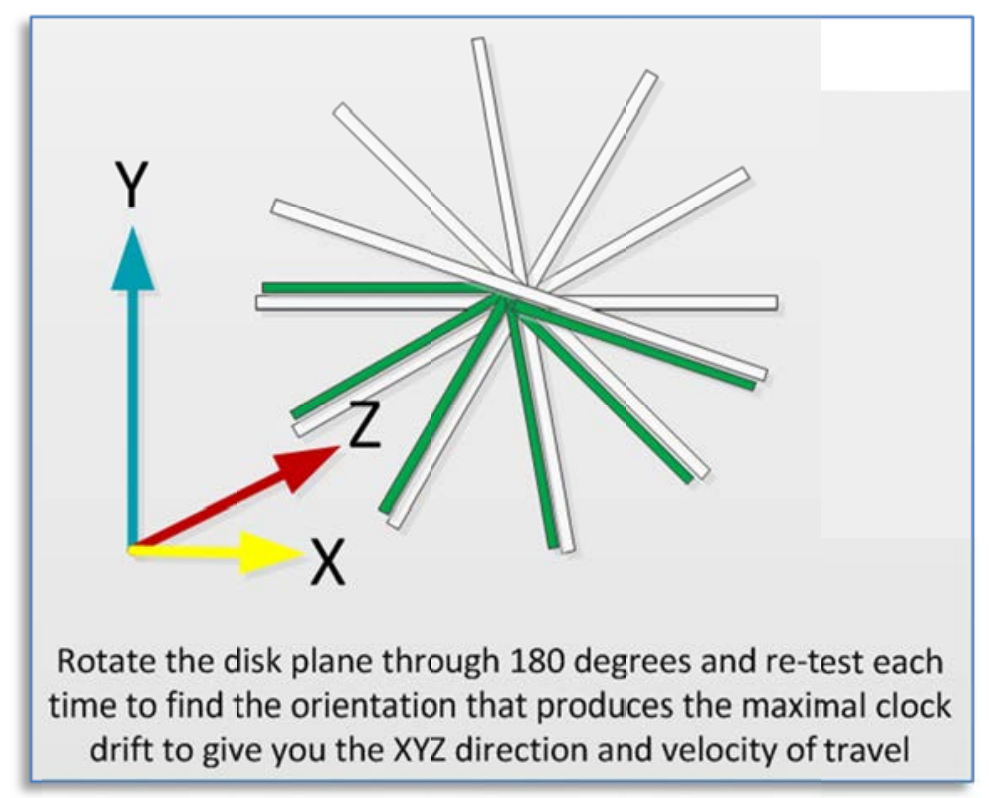

Figure 12.

\section{A spinning disk, rotated 180 degrees through a $Z$ spin axis}

Note: changing the plane of orientation of a test device on Earth from anything other than flat leads to clock synchronization issues, where the time frequency of one clock is different than the time frequency of the other clock due to General Relativity effects. For example, if your clocks are fixed to a vertical Ferris wheel, the clock tick frequency rate between the two clocks would only be synchronized at positions 3:00 and 9:00 O'clock (the point of equal gravitational attraction).

The ideal use for a rotating disk device that has three degrees of rotation (through the $\mathrm{X} / \mathrm{Y} / \mathrm{Z}$ axis's) would be as a navigation device on an inter-stellar spaceship, where independent of any external observations, you can determine the speed, and direction of travel of your spaceship relative to absolute spacetime. This same device could also provide a means for identifying and measuring dead stop in absolute spacetime, where the speed of one-way light is the same in all directions. 


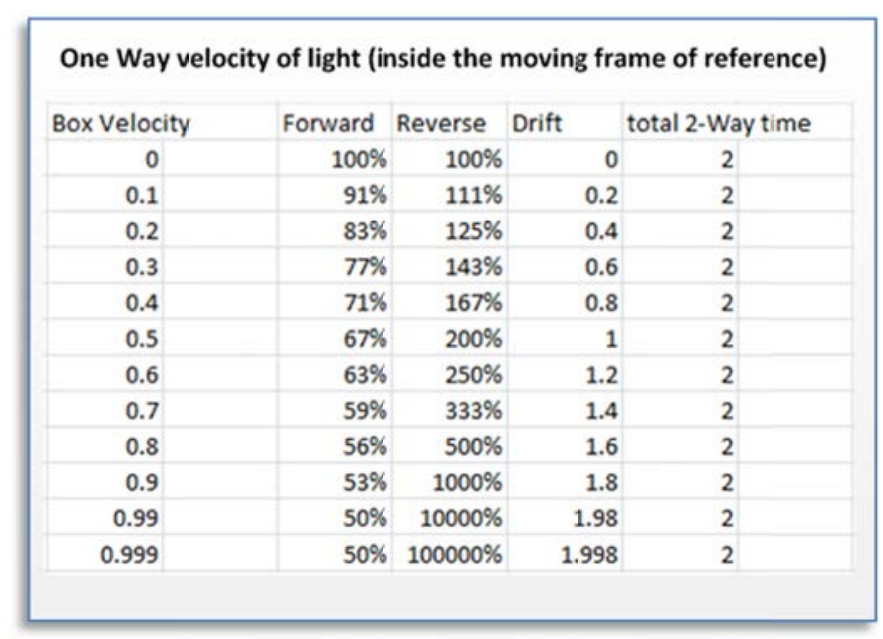

Figure 13.

At $99.9 \%$ the speed of light, as measured in the moving frame of reference, the forward one-way speed of light is $50 \%$ the speed of light, the reverse one-way speed of light is $100,000 \%$ the speed of light. The drift range between fastest and slowest measured times is 1.998 seconds. The total two-way time to traverse the disk is 2 seconds.

\section{A Practical Test for Determining the One-Way Speed of Light.}

The surface of the Earth at the equator is moving at $0.460 \mathrm{KM} / \mathrm{Sec}$. Geostationary satellites orbit the Earth at 3 $\mathrm{KM} / \mathrm{Sec}$. The Earth orbits the Sun at $30 \mathrm{KM} / \mathrm{Sec}$. The solar system orbits the Milky Way galaxy at roughly 200 $\mathrm{KM} / \mathrm{Sec}$, and the Milky Way galaxy is headed towards the direction of Centaurus at more than $600 \mathrm{KM} / \mathrm{Sec}$, and the universe is expanding at 67 kilometers per second / parsec.

The velocity of the Earth orbiting the Sun is fast enough to detect relativistic effects with today's atomic clocks. The other velocities due to the orbit of the Sun in the galaxy, and the movement of the galaxy through the cosmos and the expansion of the universe will undoubtedly affect the predicted hypothetical results, and will likely increase the predicted drift amounts.

Two things you need to know before we proceed: Viewed from above the North Pole, the Earth rotates counter-clockwise. We know this because the Sun rises in the East. Second, viewed from the North Pole, the Earth orbits the Sun in a counter-clockwise direction. We know this because we can track Earth's location relative to the other eight planets in the solar system that also orbit the Sun counter-clockwise (Including Pluto).

\subsection{The Experimental Set-Up is as Follows:}

A simple experimental set-up to measure the one-way speed of light can be done by placing two clocks on separate platforms, lined up in a West-East line-of-sight orientation, at a distance of $10 \mathrm{~K}$ apart. The clocks have the same time accuracy, but are not time synchronized or frequency synchronized. Laser light is fired from the West clock to the East clock at exactly one second tick mark intervals as defined by the West clock. The East clock tracks the local time that the West clock laser light was received, and monitors for time drift around the one second tick mark.

The clocks are next adjusted and confirmed to both be running at the same time frequency. (How this is done is described further down in this paper). Once everything is stable, the measured drift value about the one second tick mark should be a sinusoidal curve that repeats every 24 hours. At any point in the sequence of measurements, the drift value measured 180 degrees earlier should match, confirming that the West and East clocks continue to have the same time accuracy. 


\section{Thought Experiment \#2:}

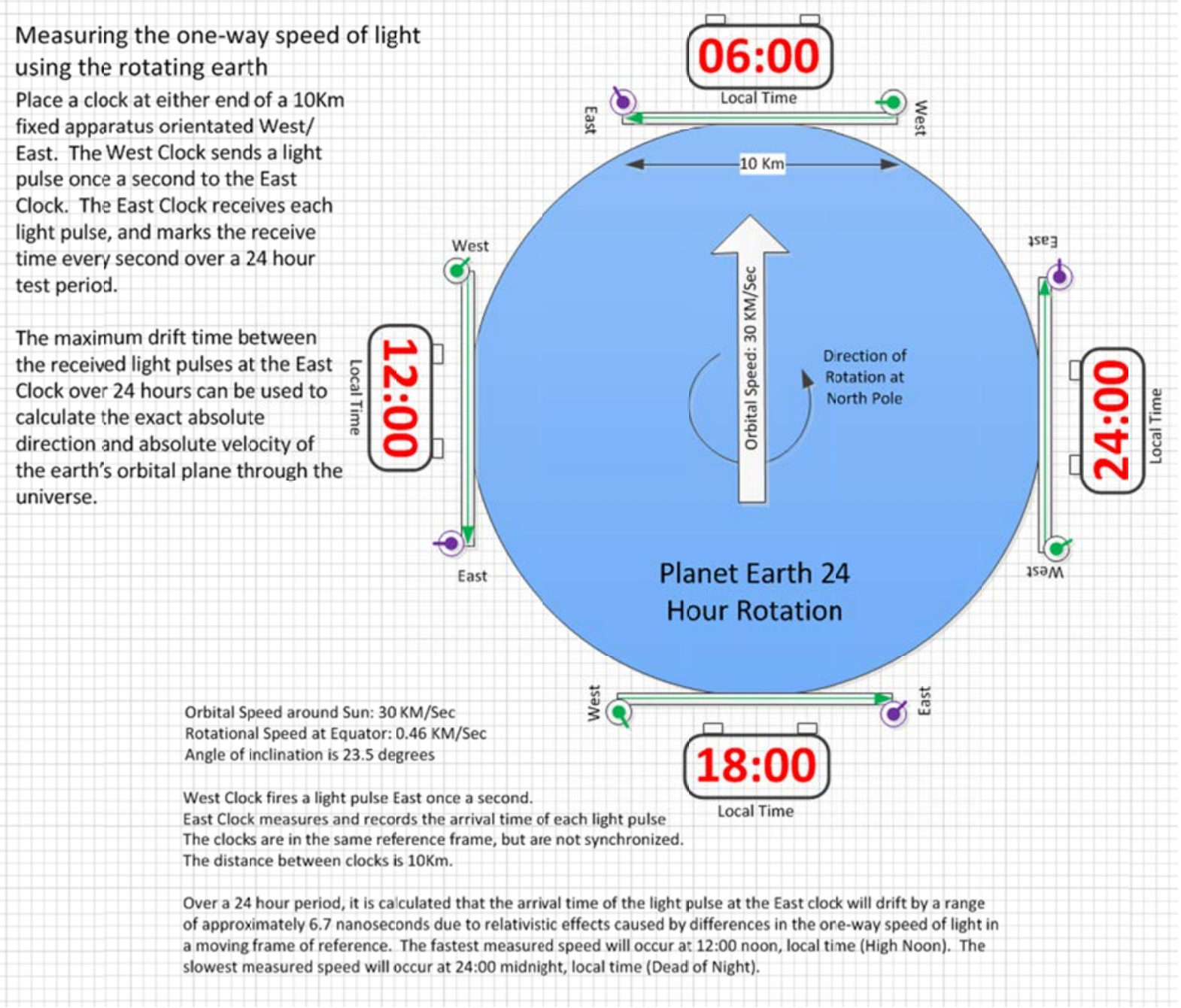

Figure 14.

The point of maximal forward speed of the fixed device occurs at two points in the day. Maximal forward speed happens when the West-East device aligns exactly with the Earth's orbital direction around the Sun. This happens during mid-day, when the Sun is highest overhead, at a point in time called High Noon. The second point of maximal forward speed occurs at the Dead of Night, 12 hours later.

Light travelling from West to East at High Noon causes the measured drift value at the East clock to go negative. This is because light is travelling in the reverse direction of Earth's orbital velocity, and in the Earth's frame of reference one-way light speed is faster than the measured two-way light speed. Another way of putting this is that light from the West arrives earlier than the one second tick mark on the East clock.

Light travelling from West to East at the Dead of Night causes the measured drift value at the East clock to go positive. This is because light is travelling in the forward direction to the Earth's orbital velocity, and in the Earth's frame of reference one-way light speed is slower than the measured two-way light speed. Putting it a different way, light from the West arrives later than the one second tick mark on the East clock.

High noon can be calculated in local time as half way between Sun-rise and Sun-set. For example, if the Sun rises at 6:45 AM, and sets at 7:27 PM, (12 hours, 42 minutes of daylight), then high noon is 6 hours and 21 minutes after sunrise, or 1:06 PM. The Dead of Night occurs exactly 12 hours later, at 01:06 AM. Note that high Noon and Dead of Night are localized reference times relative to a longitude and latitude position on the Earth's surface, independent of local time values, time zones, or daylight savings settings. 


\subsection{Accounting for the Earth's Tilt}

Because the Earth is tilted 23.6 degrees, the West/East latitude line segment may be up to 23.6 degrees off dead centre when compared to the orientation of the orbital plane. The East West plane and the Earth's orbit plane are exactly parallel only twice a year, once during the summer solstice, and a second time during the winter solstice. As the two planes become parallel, maximum speed of the West-East device in the forward direction is created. The one-way speed of light from the West at High-Noon is therefore maximal during the two solstices.

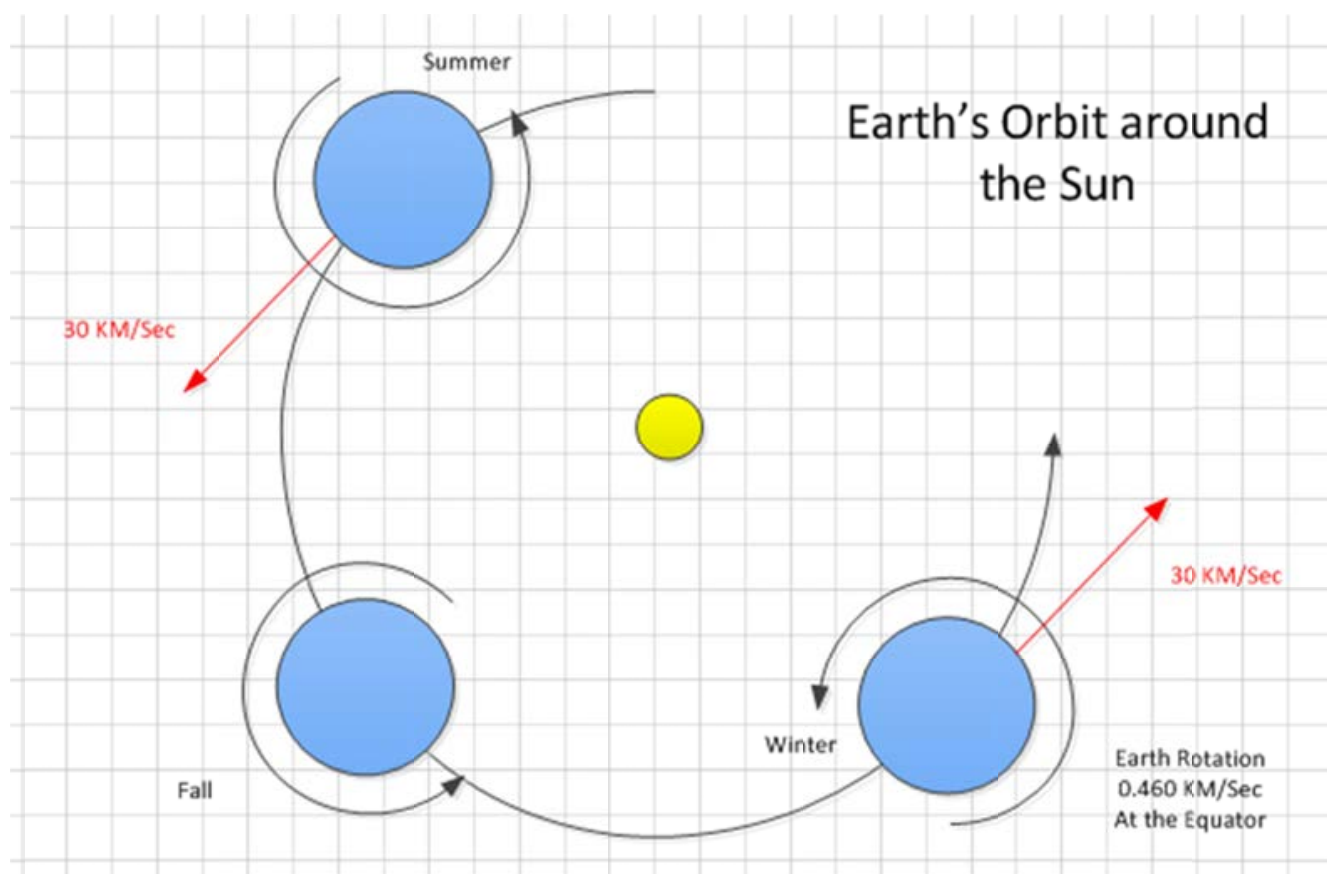

Figure 15.

If you do the experiment during one of the solstices, measured over a $10 \mathrm{Km}$ distance, the maximal drift value between the fastest and slowest measured one-way speed of light over 24 hours is calculated to be $6.6759 \mathrm{E}-09$ seconds. The fastest registered drift speed (ahead of the one second tick mark) occurs during the High Noon measurement, and is calculated to be 3.33795E-09 seconds. (3.3 Nanoseconds).

Using the drift numbers, and knowing the 2-way speed of light over that same distance, you can calculate that the speed of light in the West-East direction at High-Noon is $100.010008 \%$ the speed of light, and in the West-East direction at the Dead of Night, light travels at $99.989994 \%$ the speed of light.

\subsection{Formulas Used}

These formulas are included in a companion spreadsheet that can be downloaded from http://one-way-lightspeed.com 


Speed of Light: $c=299792 \mathrm{Km} / \mathrm{Sec}$
Speed of the Box: $V=30 \mathrm{Km} / \mathrm{Sec}$
Length of Box: $L=10 \mathrm{Km}$
Time Dilation: $\mathrm{D}=\frac{\mathrm{C}}{\sqrt{\mathrm{C}^{2}-\mathrm{V}^{2}}}$
Relativistic Length: $\mathrm{R}=\mathrm{L}^{*} 1 / \mathrm{D}$
Absolute time to go from West to East:
$\mathrm{t}=\mathrm{R} /(\mathrm{c}-\mathrm{V})$
Absolute time to go from West to East:
$\mathrm{t} 2=\mathrm{R} /(\mathrm{c}+\mathrm{V})$
Photon distance travelled West to East:
$\mathrm{P}=\mathrm{t} * \mathrm{c}$
Relativistic Measurements
(inside the box)
Length of Box: $\mathrm{L}$
Travel Time: $\mathrm{t}^{\prime}=\mathrm{t} / \mathrm{D}$
Speed of Light $\mathrm{c}^{\prime}=\mathrm{L} / \mathrm{t}^{\prime}$
$\%$ speed of $\mathrm{c}: \%=\mathrm{c}^{\prime} / \mathrm{c}$

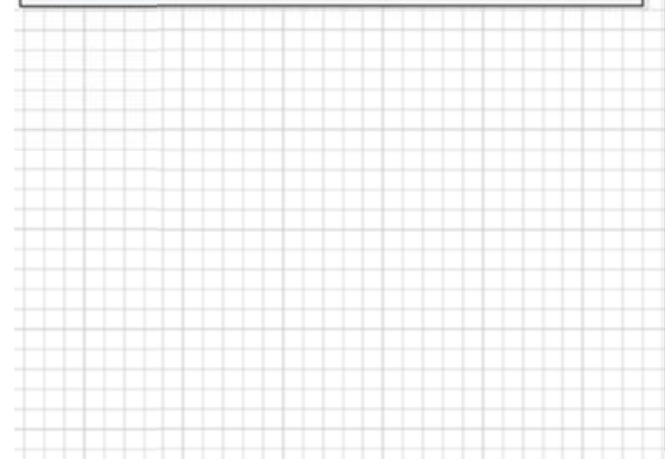

\subsection{I know what I don't know}

4.4 know what I don't know

These calculated numbers ignore the direction of the Earth is moving through absolute space time, as it travels with the Sun around the Milky Way galaxy, and as the galaxy itself drifts through the cosmos.

\section{A Practical Method for Measuring the Earth's Motion through Absolute Spacetime}

The ideal measuring device for determining the speed and direction of Earth through absolute spacetime would be two orbiting satellites, both orbiting at the same height and speed over the Earth, always at the same multiple-kilometre distance from each other, in a polar orbit that regresses a couple of degrees each complete orbit, tracing the lines of longitude around the Earth. As the satellites orbit the Earth, the linear orientation of the measurement plane changes over time, providing an opportunity to test every disk plane orientation through 360 degrees. In this fashion, the absolute direction of motion of the Earth through space time, and the absolute velocity can be determined.

The maximal XYZ Drift value measured by the satellites identifies the XYZ direction of Earth's motion through absolute space. The ratio of the two one-way light speeds measured at that XYZ point indicates the Earth's absolute speed (simple reverse lookup in a table).

The absolute speed and direction of Earth though space varies over 365 days as the Earth orbits the Sun. 


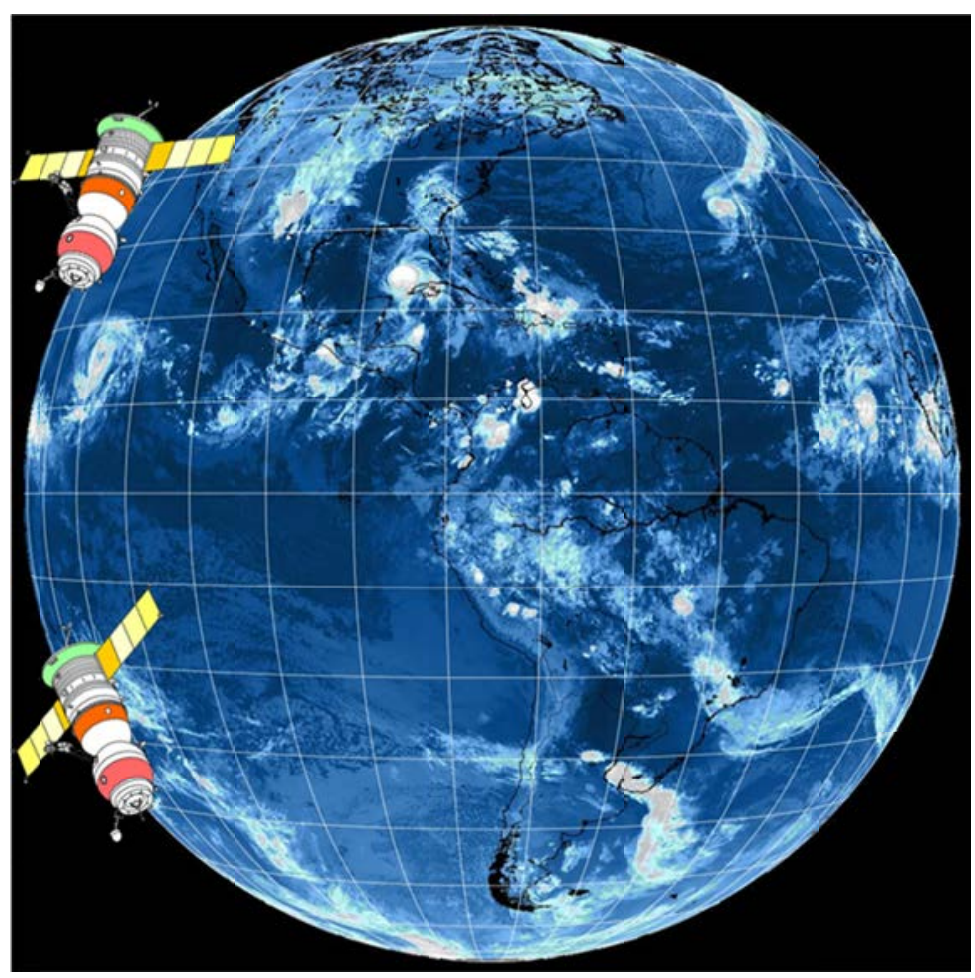

Figure 17.

\section{A Method and Apparatus For Synchronizing Two Clocks}

Measuring the one-way speed of light requires first producing a provably valid set of synchronized clocks.

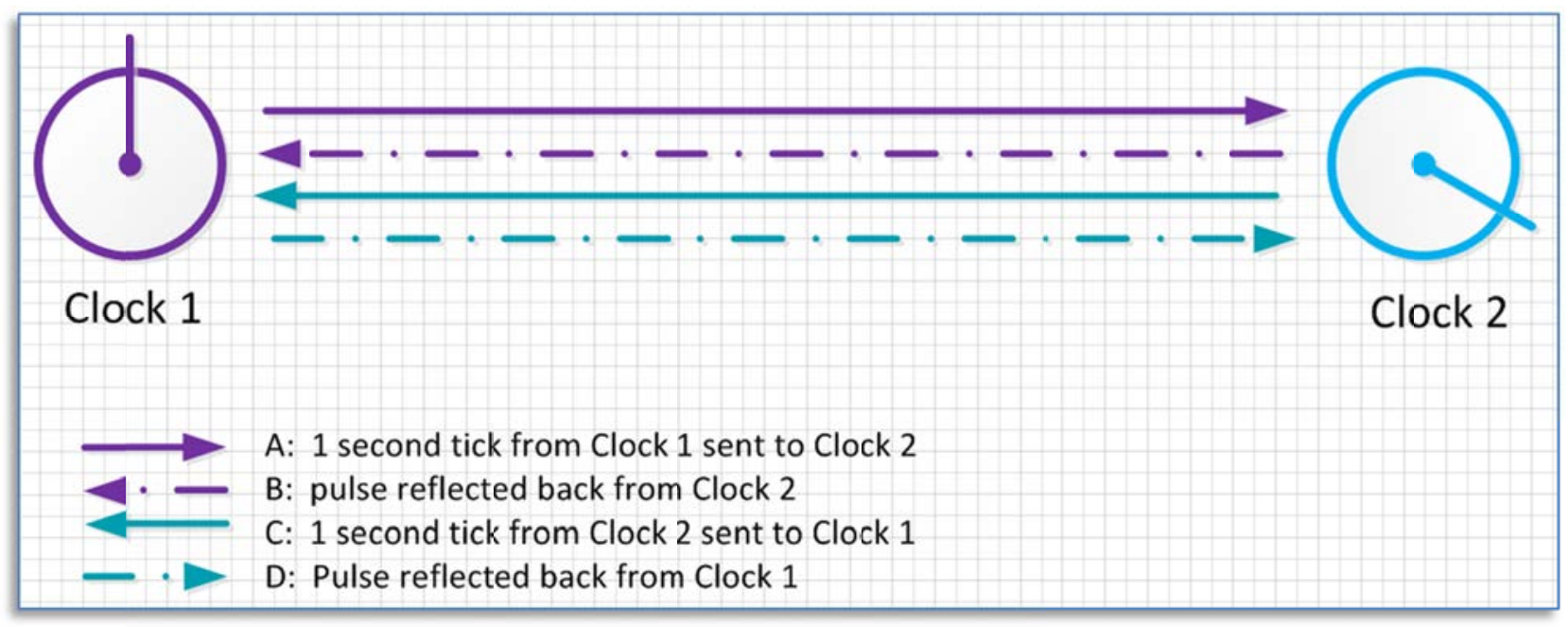

Figure 18.

6.1 Here's an algorithm for synchronizing two clocks:

1) Acquire two atomic clocks accurate to 10E-13 seconds. Call these clocks Clock 1 and Clock 2.

2) The clocks are set up to send and receive light pulses.

3) The clocks are placed 10K apart (or more) on Earth.

4) Each clock has 4 functions:

a. Send out a one second tick signal EVERY Second 
b. Send out a response signal every time you receive an incoming tick signal

c. Receive a 1-way tick signal EVERY Second

d. Receive a 2-way response signal every '2-way speed of light' interval after sending out a tick signal.

5) Over the course of 24 hours (one rotation of the Earth) plot a 1-way Drift Chart, and a 2-way Speed Chart.

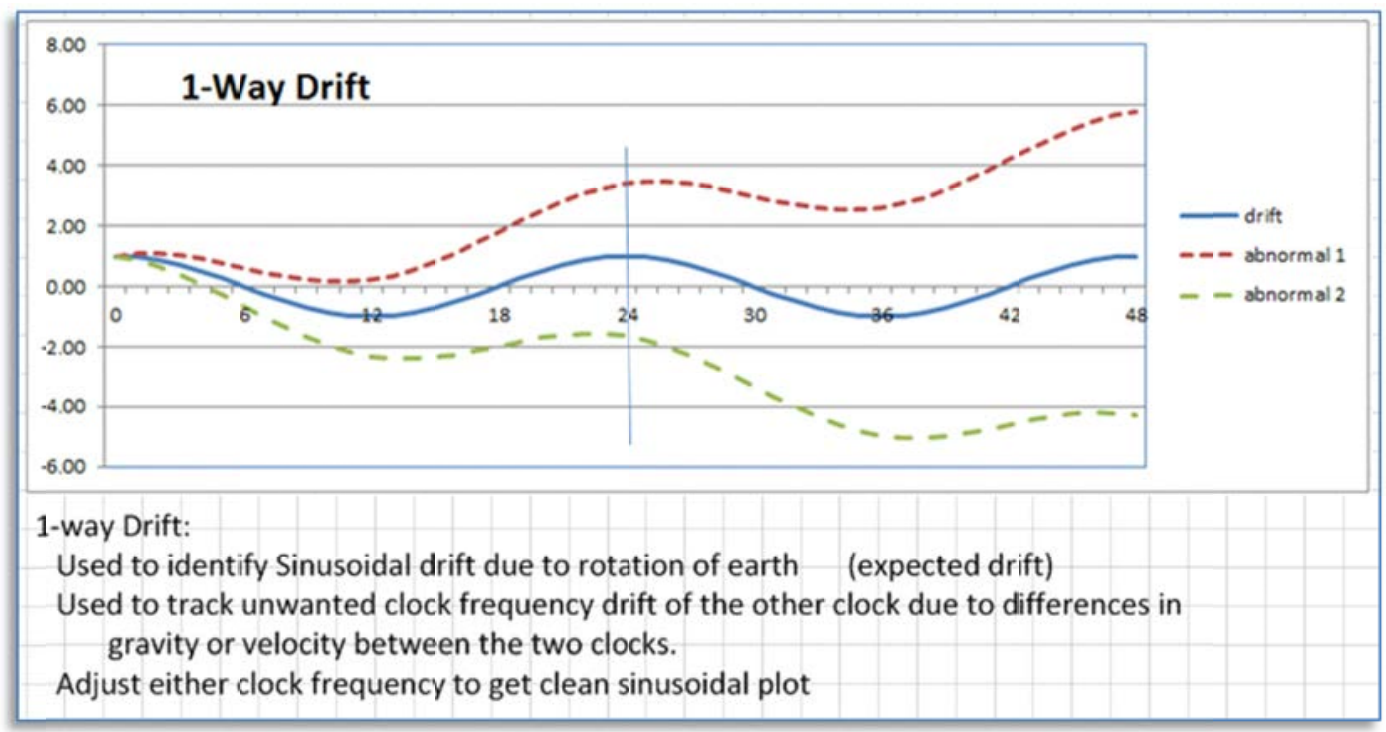

Figure 19a.

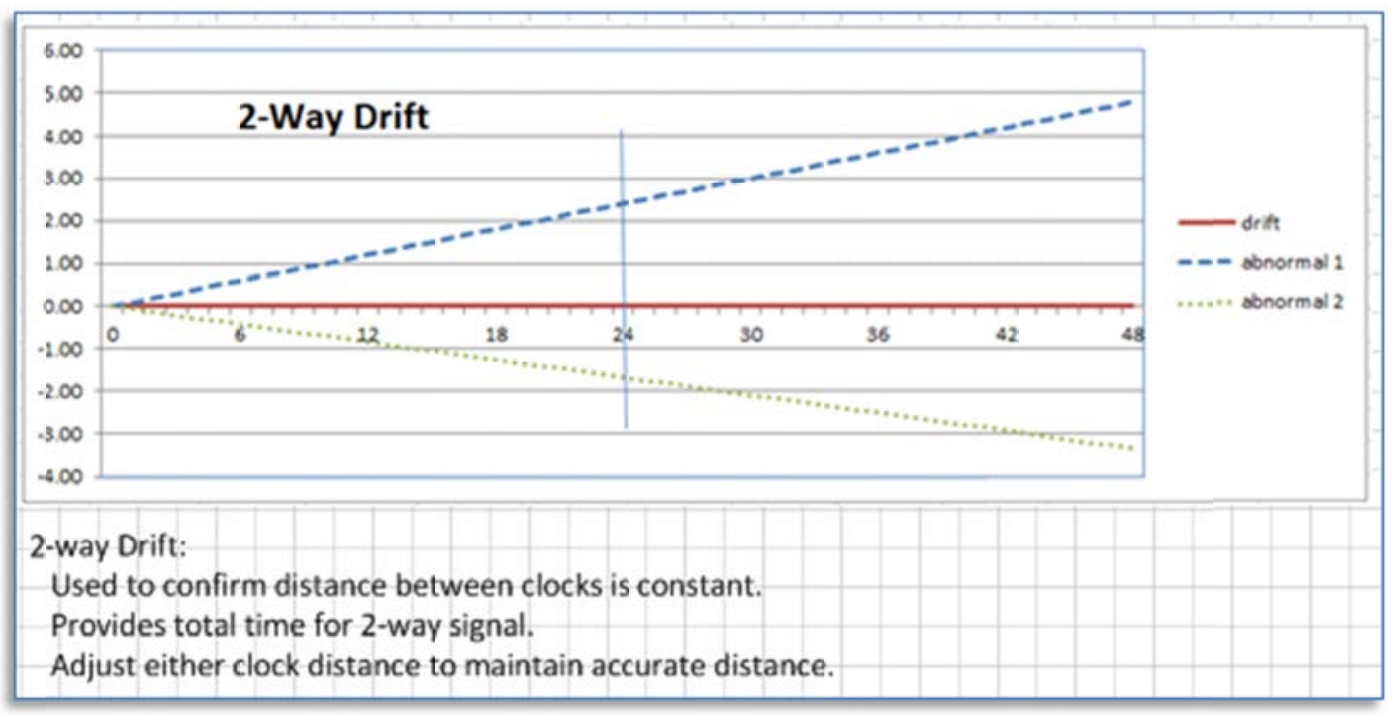

Figure 19b.

6) Review the charts, and correct for identified problems.

If the Drift Chart is not sinusoidal with an axis $\mathrm{Y}=0$, then look for and correct the clock frequency problem:

If Clock 1 is faster than Clock 2, the drift pattern on Clock 1 will trend up, and the drift pattern on Clock 2 will trend down. The drift pattern on Clock 1 trends up because the delay between the expected time of the signal and the arrival of the 1 second tick signal keeps going up (getting longer). The drift 
pattern on Clock 2 trends down because the 1 second tick signal keeps arriving earlier than expected, so the delay goes negative.

Adjust either Clock 1 or Clock 2 to synchronize the timing signal.

After frequency synchronization, the 24 hour 1-way Drift charts for both clocks should have an axis $\mathrm{Y}=0$, but be perfectly phase shifted (upside down one to the other).

If the 2-way Speed Chart is not a flat line, look for and correct the following problems:

Confirm that the distance between the two clocks has not changed. Look for Field effects that might be affecting the apparatus, including gravitation changes, acceleration changes, velocity changes,

electromagnetic issues, transmission medium issues, force, centripetal motion, temperature, hardware problems, etc.

After completing the test, the 24 hour Two-Way Speed Chart should be a flat line, and be the same for both clocks.

7) Initiate the one-time synchronization sequence.

Define Clock 1 to be the master clock.

Send time from Clock 1 to Clock 2 attached to the tick signal.

On receipt of Clock 1 timing signal, Clock 2 sets it's time to be:

Clock 1 time

+ Calculated nanosecond delay required to process the signal

$+1 / 2$ total time for 2-Way light as measured by Clock 2

+/- The measured drift value for that particular point in time of the Earth's rotation.

8) Clock 1 continues to monitor Clock 2's time

Send the time from Clock 2 back to Clock 1 in the tick signal.

On receipt of Clock 2 timing signal, Clock 1 calculates Clock 2 time as follows:

+ Calculated nanosecond delay required to process the signal

$+1 / 2$ total time for 2 -way light as measured by Clock 1

+ - The measured drift value for that particular point in time of the Earth's rotation.

9) FINALLY, Clock 1 continues to compare Clock 1 time, and calculated Clock 2 time, saving the difference in a separate synchronization drift table. If the clocks are perfectly synchronized, this value should always be zero.

Note: This is the Einstein synchronisation technique, modified by adding/subtracting measured relativistic one-way clock drift for that particular point in time of the Earth's rotation and orbit.

6.2 Using Synchronized Clocks to Measure One-way Speed

When using the synchronized clocks to measure the one way speed of light, subtract the start time from the end time, then adjust the resultant measured value by adding/subtracting the plotted relativistic one-way clock drift value for that particular point in time of the Earth's rotation and orbit.

\subsection{Measuring the Speed of a Neutrino}

If you have two perfectly synchronized clocks, and a known distance between them, and you measure the speed of the neutrino on Monday morning at 6:00 AM in a West-East direction, and then check the Start and Stop clocks, the result shows you that the neutrino travelled at the speed of light.

Using the exact same experimental set-up, on Tuesday at 1:06 PM, you do a second test, check the clocks, and the result shows you that the neutrino travelled at $100.010008 \%$ the speed of light. What do you do? Go out and re-write the laws of physics, or do you re-check the clocks?

You re-check the clocks.

The third test is carried out at 6:00 PM on Thursday. The speed of the neutrino is back to being the speed of light. What do you do now? Publish? 
The answer is you need to adjust the measured one-way speed value for the relativistic affect caused by the movement of the Earth through spacetime. This is a measured value calculated as you synchronized your clocks. The values cycle through a 24 hour period, and a 12 month period.

6.4 My educated guess is that...

At High Noon, neutrinos travel in the West-East direction faster than the speed of two-way light, but after adjusting for one-way relativity effects, they still travel at the speed of light.

At the Dead of Night, neutrinos travel in the West-East direction slower than the speed of two-way light, but after adjusting for one-way relativity effects, they still travel at the speed of light.

\section{In Conclusion}

There is some fuzzy thinking left over from 1905 that I think needs to be cleaned up.

For starters, the statement that "Light travels at the same speed in all frames of reference" actually has a double or triple meaning that needs to be clarified.

1) There is only one speed of light. Independent of all frames of reference, this is a universal constant value that never changes.

2) The two-way measured speed of light in a moving frame of reference is also a constant, and matches the universal constant speed of light due to relativistic effects accounted for by the Lorentz factor. In a moving frame of reference, time slows down, and the frame compresses in the direction of motion.

3) Measurements of light speed are always done two-way because no one has figured out a way to synchronize clocks to accurately and reproducibly measure one-way speed of light. Any light speed reference in scientific literature should be modified to say: "The two-way speed of light...." This is an important clarification, as it impacts the definition of the meter itself.

4) Finally, there is a distinct possibility, but not yet a proven fact, that the one-way speed of light in a moving frame of reference is NOT a constant.

The concept of Simultaneity is also fuzzy, as it's actually a little difficult to construct a thought experiment where the observer visualizes seeing a lightning strike 'event' before they see the lightning strike 'photons'. At best, all you can do is work backwards after seeing the photons, and surmise as to what time and place in space the event took place. This approach hardly leads to a strong understanding of the underlying behaviour of Simultaneity.

Defining a fixed frame of reference for time and space to plot light behaviour provides a starting point for identifying and resolving Simultaneity logic errors. I think it's fairly obvious that if a lightning event occurs somewhere in space within one frame of reference, then that event should be considered to occur simultaneously in all other overlapping frames of reference. In other words, there isn't a skip in time between a single event occurring in one frame of reference, and a second equivalent event occurring inside a second overlapping frame of reference at some different point in time. Otherwise, to my way of thinking, there's a logical hole to be exploited, and time travel should be possible. There's also the issue of conservation of energy to be considered.

I'm not arguing with the conclusions that have already been drawn with regard to Simultaneity, but I think it's important to point out that the explanation given for the effect may be wrong. The logic problems are resolved when you introduce the possibility that the one-way speed of light is not a constant, but instead behaves Relativistically.

The one-way speed of light can be 1000's of times faster than the two-way speed of light in a frame of reference travelling at greater than $90 \%$ the speed of light.

Neutrinos can travel at greater than the two-way speed of light if they are travelling in the opposite direction of the frame of reference's direction of motion. To be able to detect neutrinos travelling faster than the speed of light, the frame of reference needs to be moving at a measurable fraction of relativistic speed (equal to or greater than $30 \mathrm{KM} / \mathrm{sec}$ ), and the neutrino needs to be moving in the opposite direction to the frame of reference movement.

There is still more that can be learned from the photon. There is such a thing as absolute space and absolute time as imagined by Newton and refuted by Einstein. The photon knows its exact velocity through absolute space. All we have to do is properly measure the one-way behaviour of a photon, and we can walk ourselves 
back to finding Dead-Stop in absolute time and space. The photon also provides us with a fixed reference from which to deduce from within a moving frame of reference, the exact direction and velocity of that frame through spacetime.

\section{The method used to measure the one-way speed of light also leads to a solution for how to synchronize two}

clocks. Einstein synchronization is adjusted to include the Relativistic clock-drift.

\section{Finally...}

I understand that the ideas presented here go against 100 years of classical thinking, but if the proposed experiments in this paper work as predicted, and the one-way speed of light is proven to be measurable and variable, and can be used to determine Dead-stop in spacetime, it does move classical theory closer to being united with quantum theory, as quantum theory requires there to be an absolute spacetime.

\section{References}

Absolute Space and Time. (2018). In Wikipedia, the free encyclopedia. https://en.wikipedia.org/wiki/Absolute_space_and_time

Relativity of simultaneity. (2018). In Wikipedia, the free encyclopedia. Retrieved from https://en.wikipedia.org/wiki/Relativity_of_simultaneity

Ahmed, M. F., Quine, B. M., Sargoytchev, S., \& Stauffer, A. D. (2012). A review of one-way and two-way experiments to test the isotropy of the speed of light. Indian Journal of Physics, 86(9), 835-848.

Ahmed, M., Quine, B. M., Pagiatakis, S., \& Stauffer, A. D. (2013). Searching for a highly unlikely frame dependent speed of light using a one-way test. arXiv preprint arXiv:1310.4466.

Ahmed, M., Quine, B. M., Pagiatakis, S., \& Stauffer, A. D. (2013). Results of a one-way experiment to test the isotropy of the speed of light. arXiv preprint arXiv:1310.1171.

Atomic Clock. (2018). In Wikipedia, the free encyclopedia. https://en.wikipedia.org/wiki/Atomic_clock

Cahill, R. T. (2006). A new light-speed anisotropy experiment: absolute motion and gravitational waves detected. Progress in Physics, 4, 73.

Cahill, R. T. (2006). A new light-speed anisotropy experiment: absolute motion and gravitational waves detected. Progress in Physics, 4, 73.

Clock Drift. (2018). In Wikipedia, the free encyclopedia. https://en.wikipedia.org/wiki/Clock_drift

Einstein Synchronisation. (2018). In Wikipedia, the free encyclopedia. https://en.wikipedia.org/wiki/Einstein_synchronisation

Emerging Technology from the arXiv. (2010). The One-Way Speed of Light Conundrum. Retrieved from https://www.technologyreview.com/s/421603/the-one-way-speed-of-light-conundrum/

Energy Equivalence. (2018). In Wikipedia, the free encyclopedia. https://en.wikipedia.org/wiki/Mass\%E2\%80\%93energy_equivalence

Fung, S. F., \& Hsieh, K. C. (1980). Is the isotropy of the speed of light a convention?. American Journal of Physics, 48(8), 654-657.

Greaves, E. D., Rodríguez, A. M., \& Ruiz-Camacho, J. (2009). A one-way speed of light experiment. American Journal of Physics, 77(10), 894-896.

Haug, E. G. (n.d.). The True One-Way Speed of Light. Retrieved from http://www.espenhaug.com/OneWaySpeedOfLight.html

Koberlein, B. (n. d. ). Burden of Proof: What if the assumption about light is wrong? What if the speed of light is actually anisotropic?. Retrieved from https://briankoberlein.com/2015/02/14/burden-proof/

Marett, D. (n.d.). One way speed of light test - is it possible?. Retrieved from http://conspiracyoflight.com/pdf/Oneway1.pdf

Nanosecond. (2018). In Wikipedia, the free encyclopedia. https://en.wikipedia.org/wiki/Nanosecond

One-way speed of light. (2018). In Wikipedia, the free encyclopedia. Retrieved from https://en.wikipedia.org/wiki/One-way_speed_of_light 
Orders of Magnitude (time). (2018). In Wikipedia, the free encyclopedia. https://en.wikipedia.org/wiki/Orders_of_magnitude_(time)

Relativistic Doppler Effect. (2018). In Wikipedia, the free encyclopedia. https://en.wikipedia.org/wiki/Relativistic_Doppler_effect

Roberts, T., \& Schleif, S. (2007). What is the experimental basis of Special Relativity?. Retrieved from http://www.edu-observatory.org/physics-faq/Relativity/SR/experiments.html

Sagnac Effect. (2018). In Wikipedia, the free encyclopedia. https://en.wikipedia.org/wiki/Sagnac_effect

Second. (2018). In Wikipedia, the free encyclopedia. https://en.wikipedia.org/wiki/Second

Spavieri, G., Quintero, J., Unnikrishnan, C. S., Gillies, G. T., Cavalleri, G., Tonni, E., \& Bosi, L. (2012). Can the one-way speed of light be used for detection of violations of the relativity principle?. Physics Letters A,

376(6-7), 795-797.

Special Relativity. (2018). In Wikipedia, the free encyclopedia. https://en.wikipedia.org/wiki/Special_relativity

Speed of Light. (2018). In Wikipedia, the free encyclopedia. https://en.wikipedia.org/wiki/Speed_of_light

The Lorentz Transform. (2018). In Wikipedia, the free encyclopedia. https://en.wikipedia.org/wiki/Lorentz_transformation

Time Dilation. (2018). In Wikipedia, the free encyclopedia. https://en.wikipedia.org/wiki/Time_dilation https://en.wikipedia.org/wiki/Length_contraction

Unit of Time. (2018). In Wikipedia, the free encyclopedia. https://en.wikipedia.org/wiki/Unit_of_time

\section{Appendix A:}

Michelson-Morley Experiment 1881, 1887:

Before the publication of Einstein's theory of special relativity, it was thought that light travelled as a wave through an invisible aether medium, much like sound propagates as a wave through air.

Assuming that the aether is stationary relative to the movement of the Earth around the Sun, then the Earth moving through the aether should affect the measured speed of light, similar to how sound travelling through air that is moving travels at a different measured speed than sound travelling through air that is still (the speed of sound, plus the speed of the travelling air). 
Accordingly, an experiment was set up in 1887 to try and measure the aether effect. This famous experiment was known as the Michelson-Morley experiment, and it spectacularly failed to prove the existence of aether.

Instead what was discovered was that the speed of light was the same no matter what direction it was measured.

The results from the Michelson-Morley experiment caused a great amount of head scratching. If the speed of light is constant in a vacuum, and you are measuring the speed of light in a very fast spaceship (like the Earth travelling around the Sun), there should be a detectable difference in behaviour when measuring the speed of light in the direction of travel compared to the speed of light at right angles to the direction of travel.

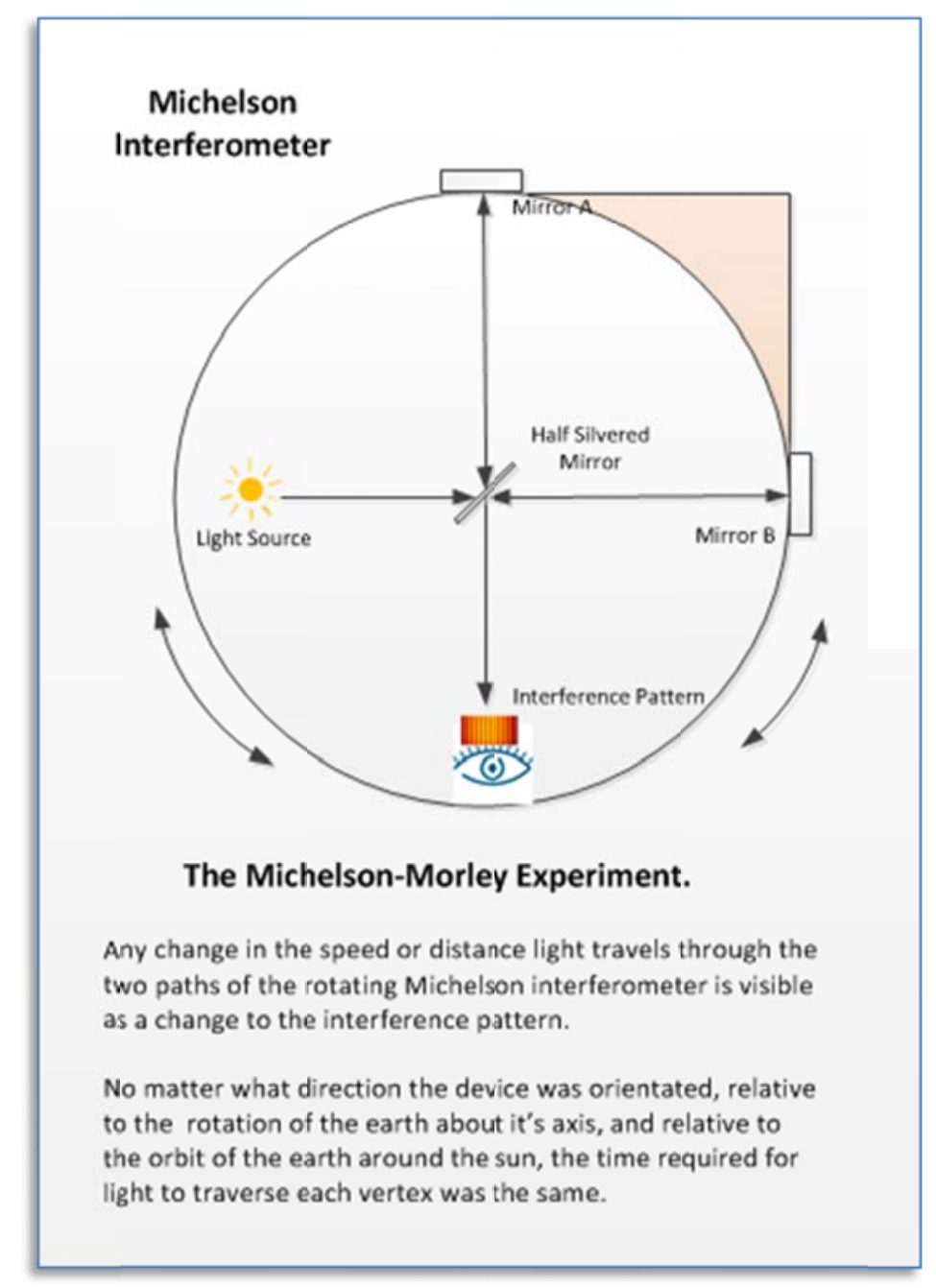

Figure 20.

\section{Appendix B:}

\section{Deriving the Lorentz Factor}

\section{B.1 Object at rest}

Shown in the diagram below is a very large box. In this box, light takes one second to travel from the bottom to the top of the box, and one second for light to travel from the left to the right of the box.

In physical terms, the width/height of the box is approximately the distance from the Earth to the Moon. 


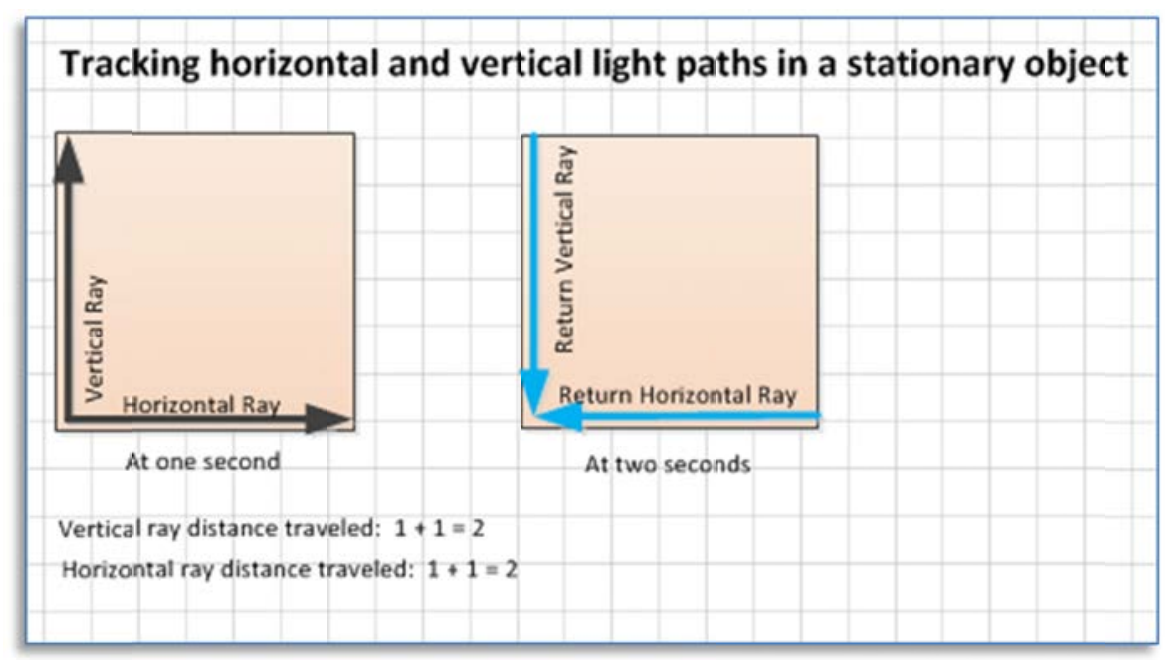

Figure 21.

In this stationary box, if you split the light at the source, sending it off in two directions at right angles to each other, the two light pulses return back to the origin of the box at exactly the same. It takes one second for the split pulse of light to reach the top of the box, and the same amount of time for the split pulse of light to reach the right hand edge of the box. It then takes one more second for the two pulses of light to be reflected back to the origin.

Total amount of elapsed time for the pulsed light beam to be sent and reflected back to the origin is exactly two seconds.

\section{B.2 Object in Motion}

Shown in the diagram below is the same box from Figure 21, but now travelling in the horizontal direction at $66 \%$ the speed of light, and at two points in time: At 1 second, and at 1.34 seconds.

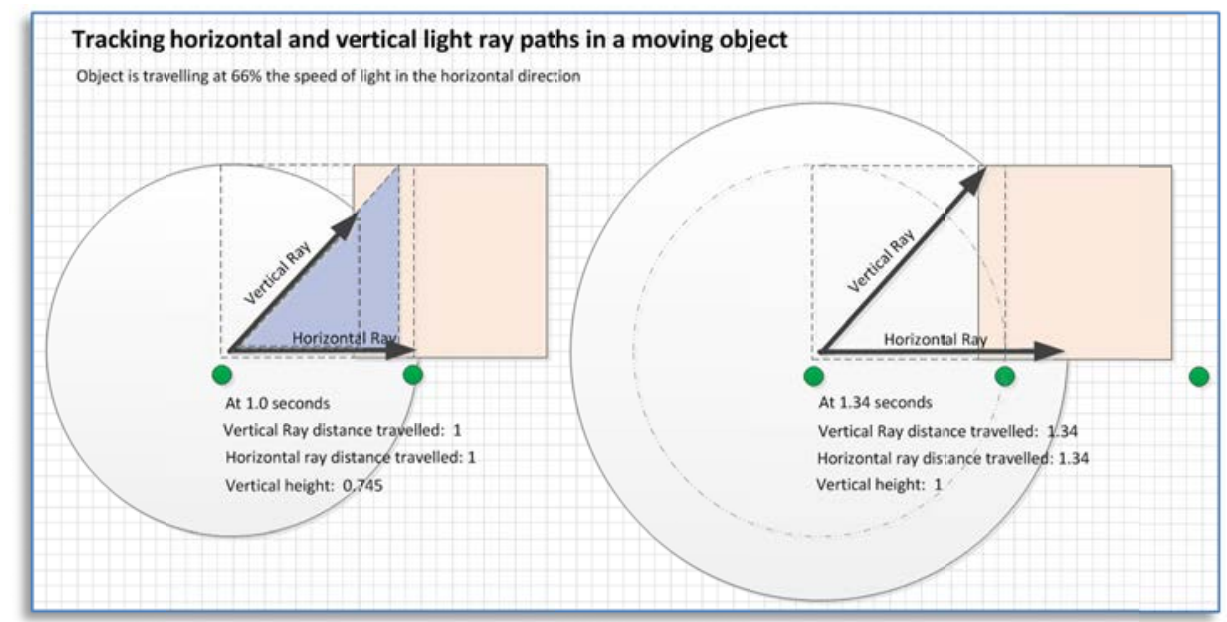

Figure 22.

If you assume that the absolute speed of light is the same in all reference frames, this means that in a moving object, light within the object still travels at the speed of light, not the speed of light PLUS the speed of the object. In other words, inside the moving object, strange things start to happen with the light.

Light rays inside the moving box and light rays outside the moving box travel at the exact same speed.

If you trace the path that a split light pulse travels in the moving box, relative to the outside frame of reference, such as two outside photons, the path traverses the right and left sides of an isosceles triangle. 
In the stationary box, the angle of the isosceles triangle is 90 . In a moving box, the angle of the isosceles triangle is dependent on the speed of the box. As speed increases, the angle of the triangle decreases, and the length of the hypotenuse increases.

In a box travelling at $66 \%$ of the speed of light, the length of the vertically orientated side of the isosceles triangle traced by the ray of light increases from 1 to 1.34 . This means that inside the box it takes 1.34 seconds for the light to reach the top of the moving box. This value can be calculated fairly easily for any box speed by comparing the relative sizes of the two similar triangles, one drawn at one second with a hypotenuse length of 1 , and the second drawn at the point the light pulse reaches the top of the box, where the length of the right angle vertical side is 1 .

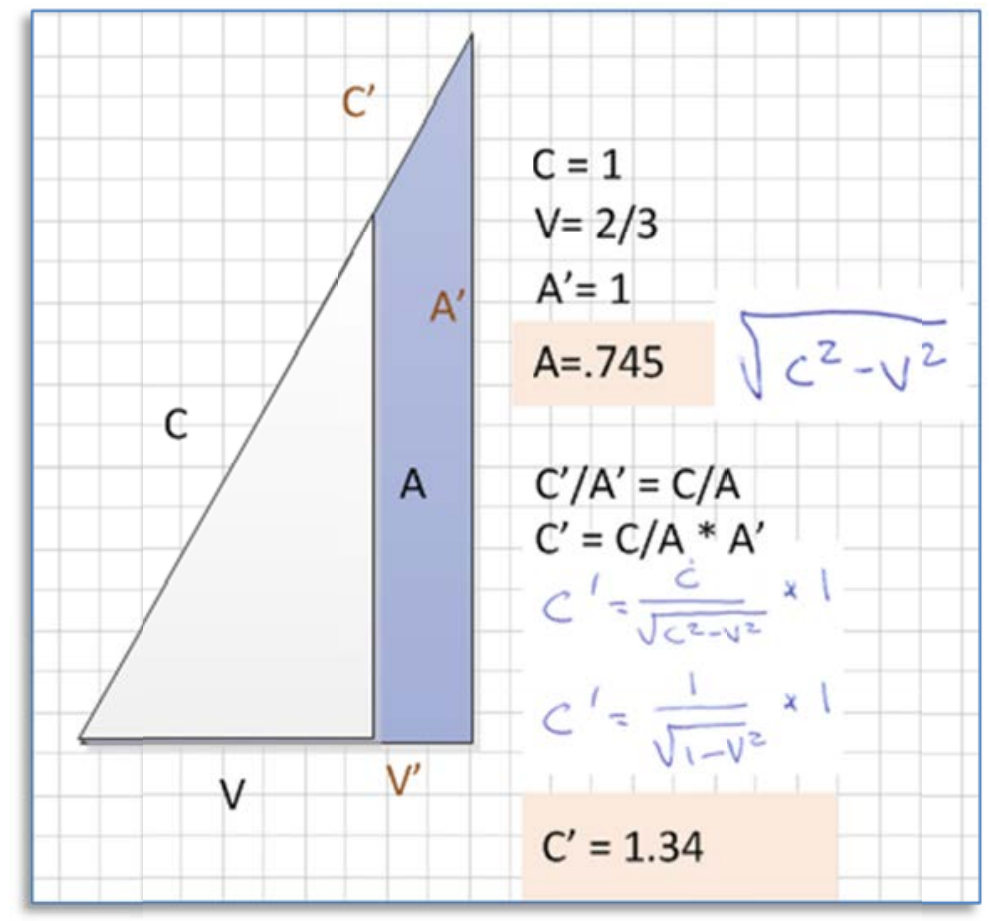

Figure 23.

We're now starting to see a problem. In the motionless box, the split light pulse reached the top and the right side of the box at exactly the same time. But, within the moving box, the vertical light pulse reaches the top of the box before the horizontal light pulse reaches the far side of the box.

Things get even more interesting if you wait a further 1.34 seconds in time. At this point 2.68 seconds have elapsed and the reflected vertical light ray reaches the XY Origin point in the moving box. However, the horizontal light ray has not yet hit the right edge of the moving box, and won't do so for another 0.32 seconds.

\section{Tracking the Return Stroke:}
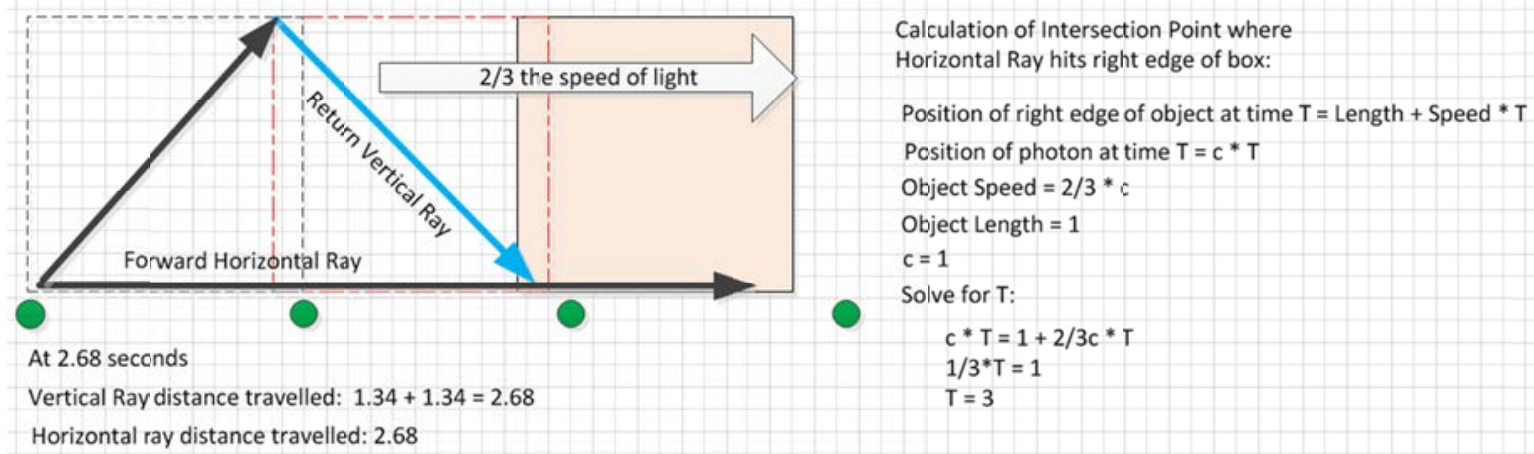

Figure 24.

So any experiment or device where the $\mathrm{X}$ and $\mathrm{Y}$ light rays return to the origin at the exact same moment in a stationary frame of reference should fail to do so when the device is moving. 
The Earth is itself an object moving through space, and as such can be used for testing out the theory of relativity.

The expectation was that the Michelson-Morley experiment would succeed in showing different light speeds depending on the direction of measurement, as the Earth is constantly in motion rotating on its axis every 24 hours, and circling the Sun every 365 days. The Sun in turn is orbiting the Milky Way Galaxy, and the Milky Way Galaxy is drifting through the universe.

It was a big deal then when the Michelson-Morley experiment failed to detect a difference in the speed of light.

One of the many theories developed to explain the results of the Michelson-Morley experiment was put forward by Hendrik Lorentz. His theory was that the length of a ruler shrinks when pointed in the direction of movement through space, as described by the Lorentz-FitzGerald contraction. The contraction of the object's length increases as the objects speed approaches the speed of light. At $90 \%$ speed of light, the objects length in the direction of travel is compressed to $44 \%$ of its original length. As an object's speed approaches the speed of light, its compressed length approaches zero.

In this way, the vertical and horizontal light rays travel different distances within the box, but still end up meeting at the origin at the same time.

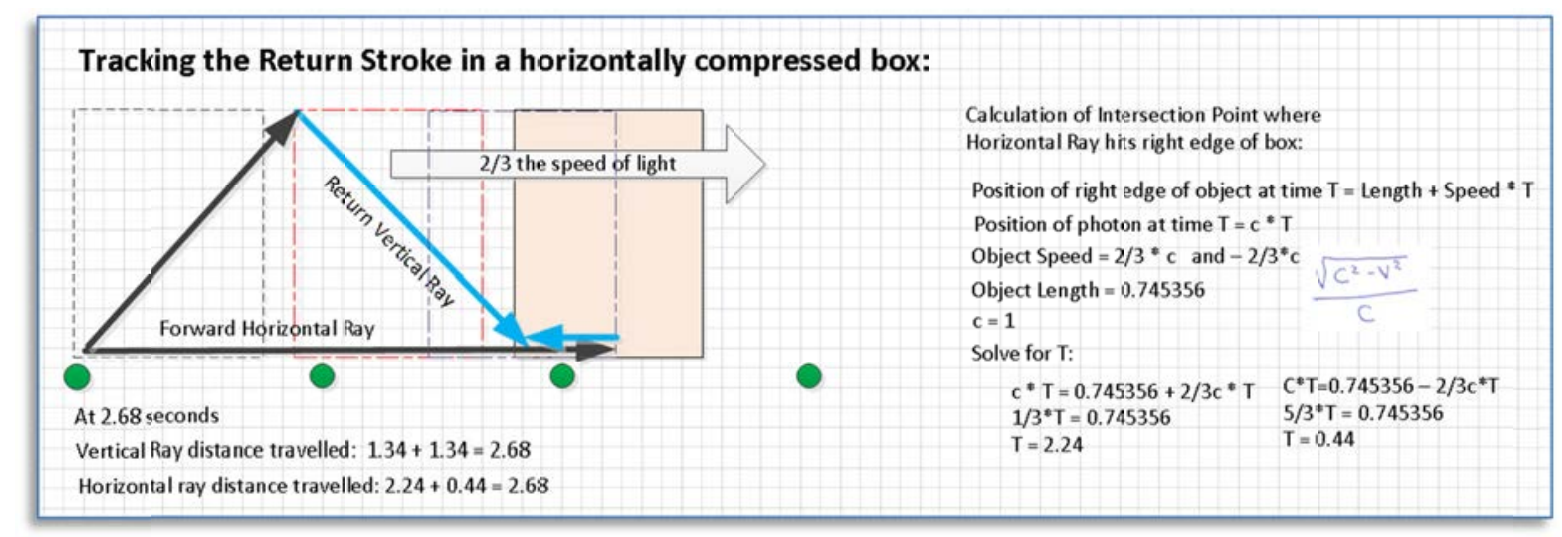

Figure 25.

To an external observer in a fixed frame of reference, if the box moving at $2 / 3$ the speed of light were to become compressed in the direction of travel to $75 \%$ of its original length, then the horizontal and vertical light rays would again converge to meet at the origin at exactly the same time. The compression factor of $75 \%$ is derived as the ratio between the 'height of the box' and the 'vertical distance light travelled in the box', otherwise known as the ratio between the opposite side and the hypotenuse of a right angled triangle, or SINE function.

The equation defining how time and space changes relativistically is called the Lorentz Factor.

I've been drawing diagrams that show the contraction relationship as a function of the ratio of the opposite side to the hypotenuse. (Same relationship as is defined by the SINE function).

How does one square this with the traditional formulation of the Lorentz factor? It turns out they are the same relationship. The Opposite/Hypotenuse relationship shown in these simple diagrams is equivalent to the Lorentz equation as it is traditionally written.

For historical reasons, it is customary to show the time dilation value as an inverted Opposite/Hypotenuse value (increasing in value as the speed increases). 


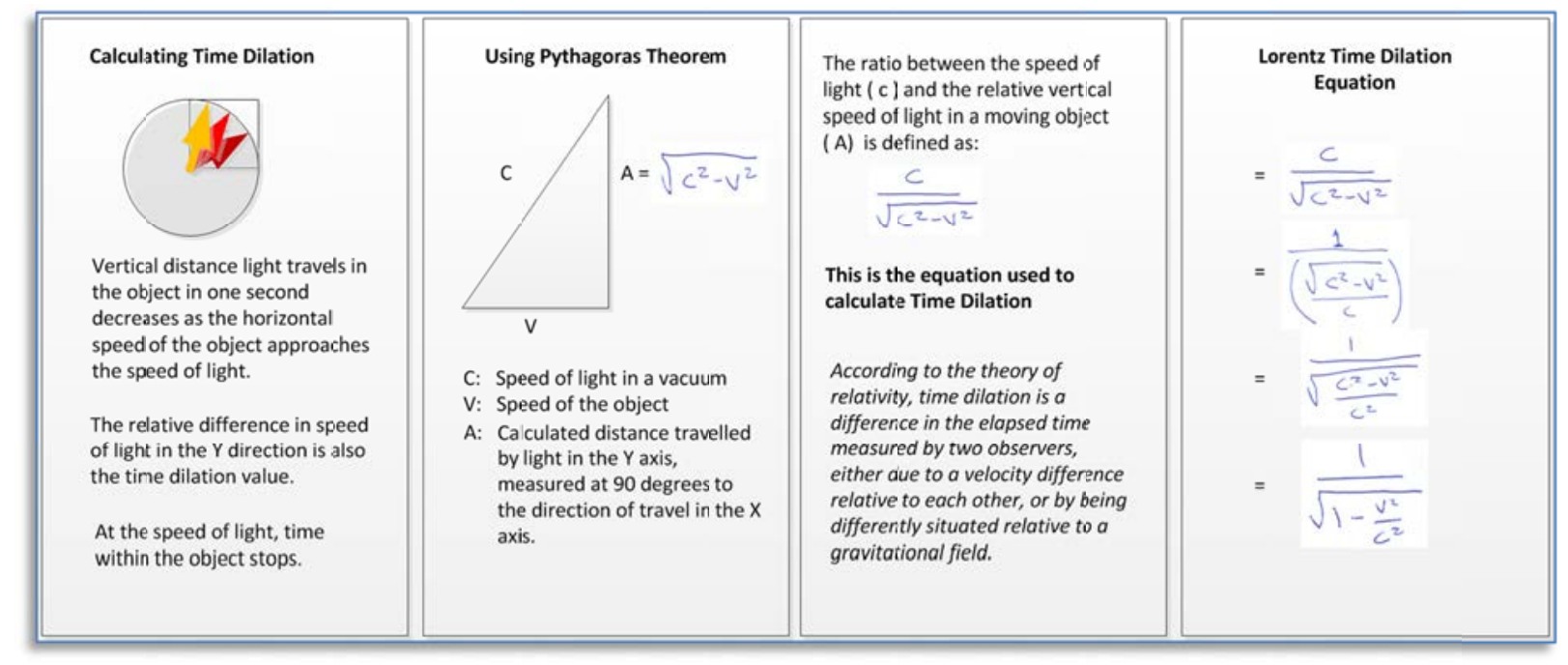

Figure 26.

A second problem then was how to account for the increase in time it takes for the light rays to return to the origin. Travelling at $2 / 3$ the speed of light, with the object's length compressed to $75 \%$ of its original size, the time it takes for the two split light pulses to simultaneously return to the origin is 2.68 seconds. In the same object at rest, the time to simultaneously return is 2 seconds.

So the second part of Hendrik Lorentz's theory was to propose that time slows down as an object increases in speed. As an objects speed approaches the speed of light, time approaches zero.

The ratio by which time slows down is the same as the compression factor, that being the ratio between the 'height of the box' and the 'vertical distance light travelled in the box'. For measuring time, the ratio is normally written down as its reciprocal; to better indicate the increasing scale of the time dilation as the objects speed approaches the speed of light.

In the external frame of reference, the time for the split light pulses to return to the origin in the box moving at $2 / 3$ the speed of light is 2.46 seconds, and the length of the box is $75 \%$ of its original size. But inside the moving box, the same speed measurement would return a value of 2 seconds, and the internal measured length and height of the box would remain as 1 light second (the distance light travels in one second).

Lorentz also disclosed how mass might increase as speed increases, and how the release of energy represents a loss of mass.

\section{B.3 In Summary:}

The Lorentz hypothesis explained the unexpected results of the Michelson-Morley experiment by stating that the speed of light is constant, but our measurements of time and space vary in a way that outside observers can detect, but someone inside the moving frame of reference would be unaware of.

1) The speed of light is constant in all frames of reference.

2) Nothing can go faster than the speed of light.

3) Time itself slows down as an object is accelerated to the speed of light.

4) Objects shrink in the direction of movement as an object accelerates to the speed of light.

5) The laws of physics behave the same in all frames of reference.

However, Lorentz still held to the notion that light waves propagate through aether, and that within that aether a resting clock would indicate the 'true time'. 


\section{Logic Conundrum Resolved using the Lorentz Factor:}

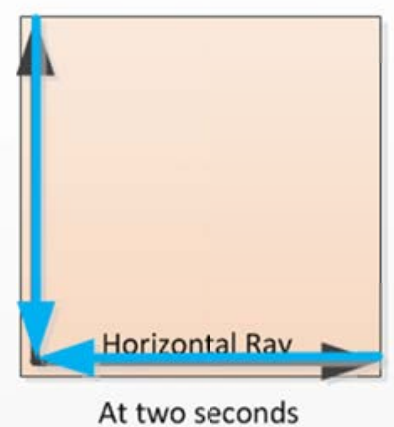

Inside the object measurements:

Elapsed Time: 2 seconds Width: 1

Height: 1

Vertical ray distance traveled: $1+1=2$

Horizontal ray distance traveled: $1+1=2$
Light traveling the $X$ and $Y$ Axis's in a square box returns to the point of origin synchronously, and at the same amount of measured time, regardless of the speed that the object is moving through space.

\section{Outside the object measurements:}

Elapsed Time: $2 * 1.34=2.68$ seconds Width: $1 / 1.34=0.745$

Height: 1

Vertical ray distance traveled: $1.34+1.34=2.68$

Horizontal ray distance traveled: $2.24+0.44=2.68$

Inside the moving box, an observer sees no change in behaviour regardless of the speed of the box through space.

At Relativistic speeds, time slows down, and the object's

Time Dilation: $\quad \frac{c}{\sqrt{c^{2}-v^{2}}}$ length contracts in the direction of movement. Length Contraction:

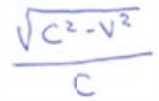

Figure 26.

\section{Appendix C:}

Relativity functions that use the Lorentz Factor 


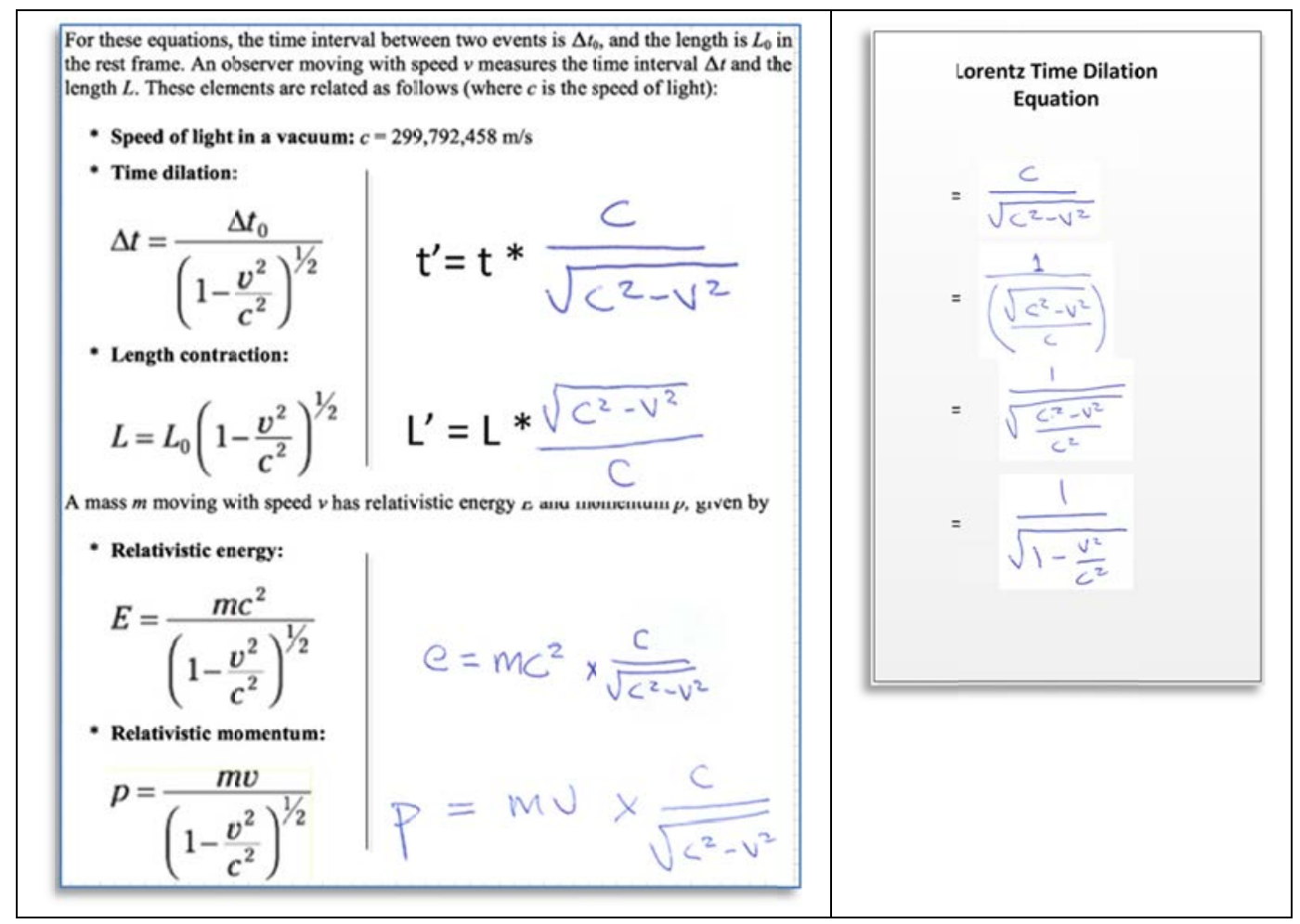

Figure 28.

http://www.dummies.com/education/science/physics/formulas-from-einsteins-theory-of-special-relativity/

\section{Appendix D:}

Time and Space are Relative

Here is example of Simultaneity where an object appears to be travelling faster than the speed of light.

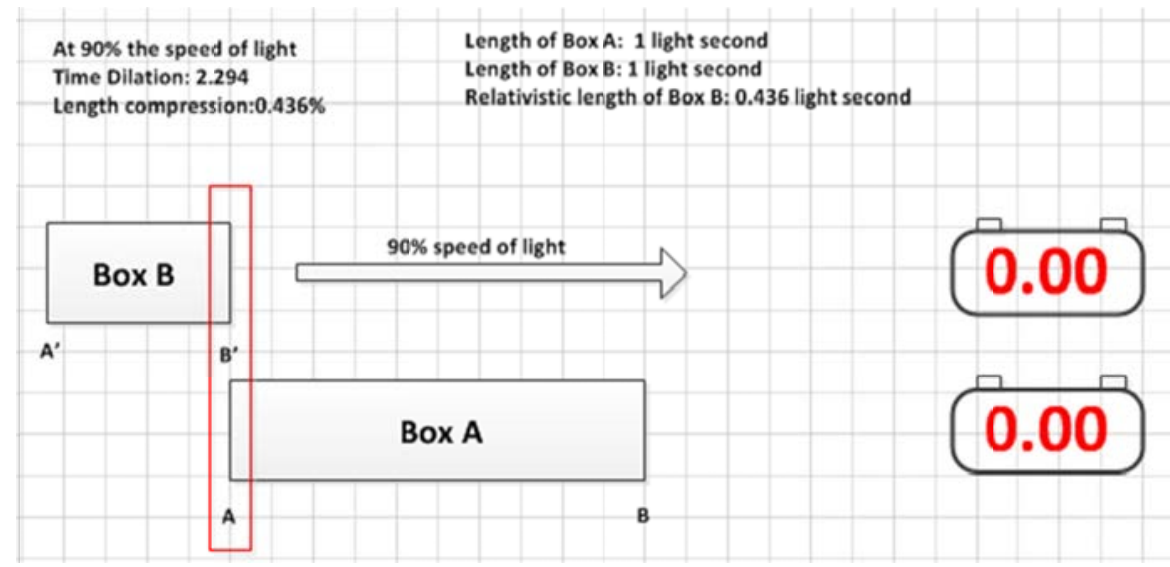

Figure 29.

Box B is travelling to the right past Box A at $90 \%$ the speed of light. Each box contains two synchronized clocks, one at each end. All four clocks are set to 0:00 the moment point B' meets up with point A. 


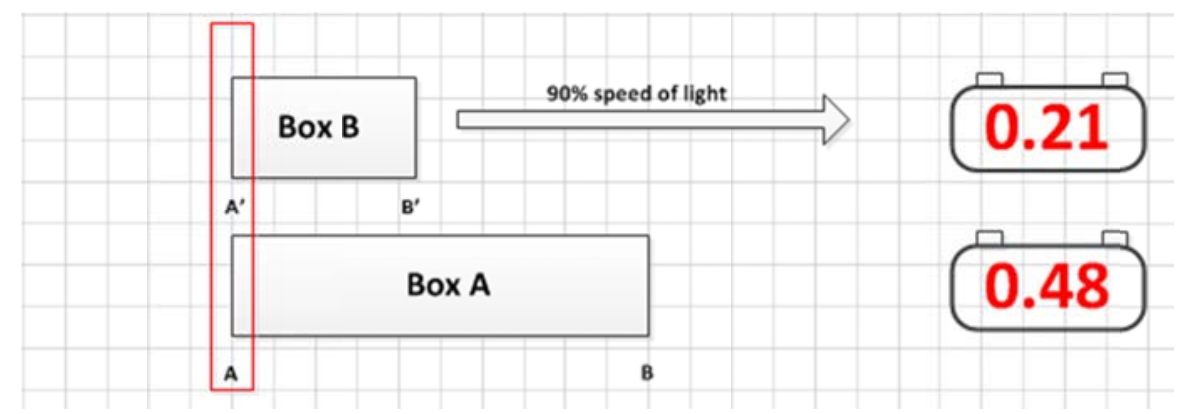

Figure 30.

Box A measures the length of Box B where A goes from B' to A' in 0.48 seconds.

Box B measures the speed of Box A where A goes from B' to A' in 0.21 seconds.

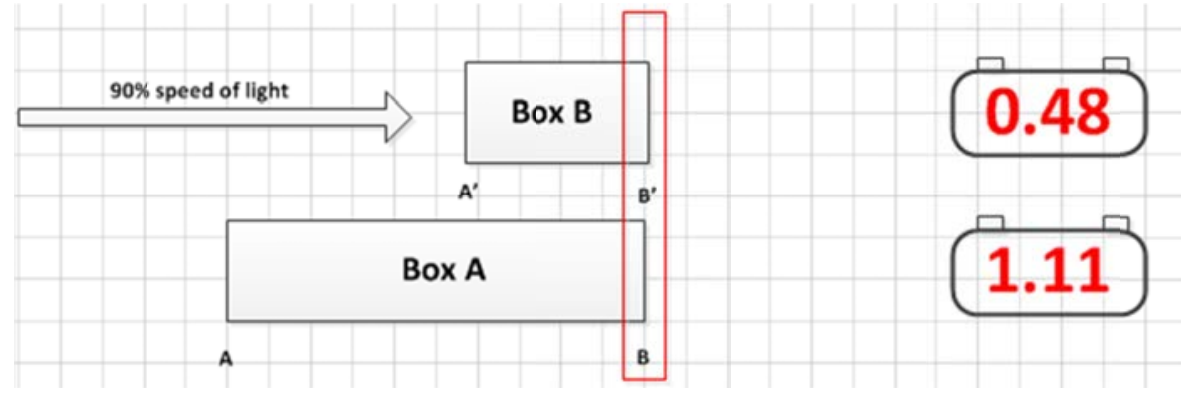

Figure 31.

Box A measures the speed of Box B where B' goes from A to B in 1.11 seconds.

Box B measures the length of Box A where B' goes from A to B in 0.48 seconds.

Box A:

The Speed of Box B is measured as the time it takes for Point B' to go from A to B.

The length of $\mathrm{AB}$ is 1 . The speed is 1 box length per $1.11 \mathrm{sec}$, or 0.9 times the speed of light.

The Length of Box B is measured as the time it takes for Point A to go from B' to A",

The time value is .48 . Box B is $0.48 * 0.9=0.436$ of a box in length.

Sees Box A as 1 in length and Box B as 0.436 in length, travelling to the right at $90 \%$ the speed of light. Box B:

The Speed of Box A is measured as the time it takes for Point A to go from B' to A'

The length of B'A' is 1 . The speed is 1 box length per 0.21 seconds, or 4.7 times the speed of light.

The Length of Box A is measured as the time it takes for Point B' to go from A to B.

The time value is .48 . Box A is $.48 * 4.7=2.29$ of a box in length.

Sees Box B as 1 in length and Box A as 2.29 in length, travelling to the left at $474 \%$ the speed of light. 


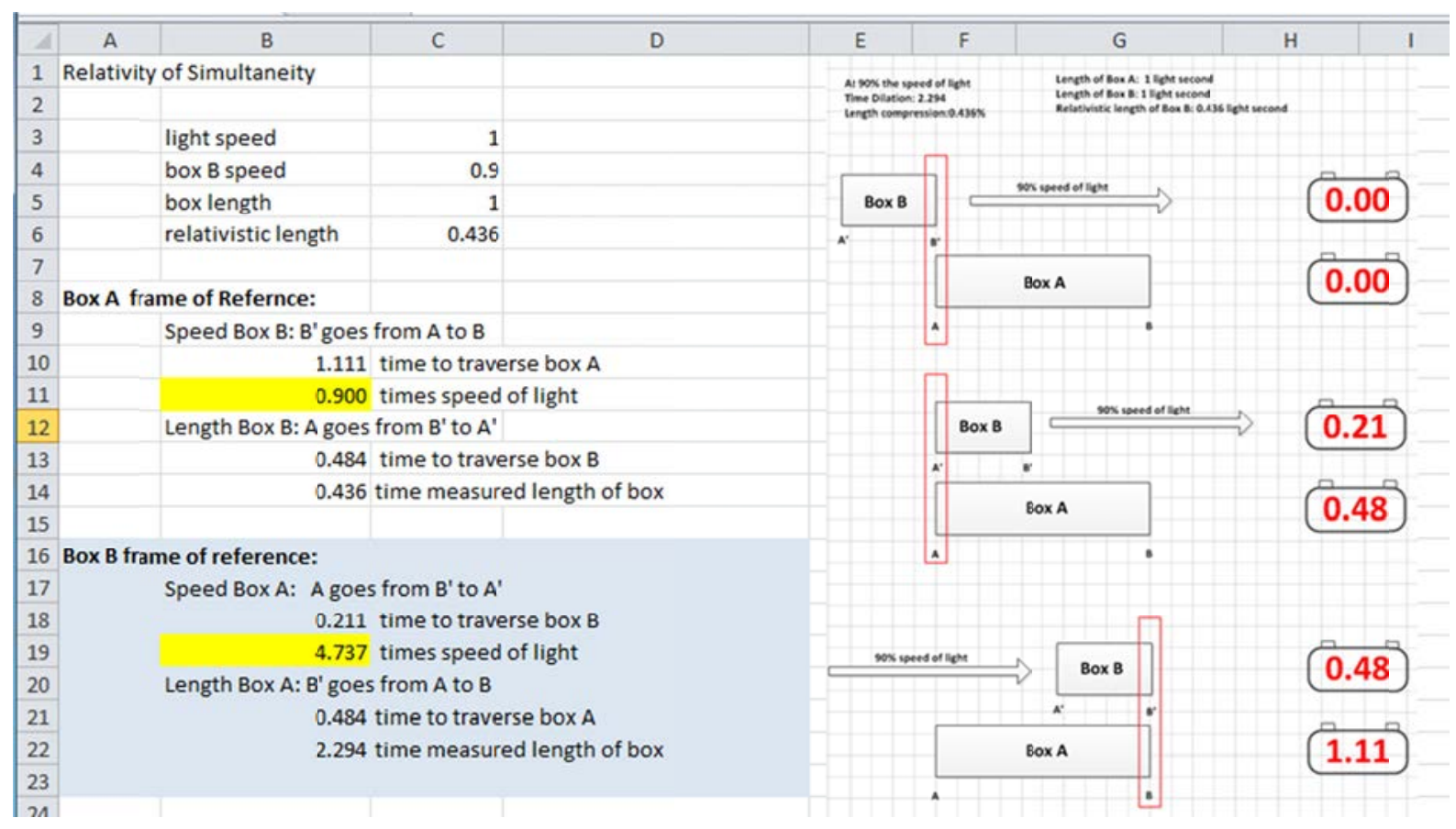

Figure 32.

\section{Appendix E}

Did a detected neutrino travel faster than the speed of light?

Quoted from Wikipedia: https://en.wikipedia.org/wiki/Neutrino

Neutrinos are created by various radioactive decays, including in beta decay of atomic nuclei or hadrons, nuclear reactions such as those that take place in the core of a star or artificially in nuclear reactors, nuclear bombs or particle accelerators, during a supernova, in the spin-down of a neutron star, or when accelerated particle beams or cosmic rays strike atoms. The majority of neutrinos in the vicinity of the Earth are from nuclear reactions in the Sun. In the vicinity of the Earth, about 65 billion $(6.5 \times 1010)$ solar neutrinos per second pass through every square centimetre perpendicular to the direction of the Sun.[11][12]

For study, neutrinos can be created artificially with nuclear reactors and particle accelerators. There is intense research activity involving neutrinos, with goals that include the determination of the three neutrino mass values, the measurement of the degree of $\mathrm{CP}$ violation in the leptonic sector (leading to leptogenesis); and searches for evidence of physics beyond the Standard Model of particle physics, such as neutrinoless double beta decay, which would be evidence for violation of lepton number conservation. Neutrinos can also be used for tomography of the interior of the Earth.[13][14]

Quoted from Wikipedia: https://en.wikipedia.org/wiki/OPERA_experiment

In September 2011, OPERA researchers observed muon neutrinos apparently travelling faster than the speed of light. In February and March 2012, OPERA researchers blamed this result on a loose fibre optic cable connecting a GPS receiver to an electronic card in a computer. On 16 March 2012, a report announced that an independent experiment in the same laboratory, also using the CNGS neutrino beam, but this time the ICARUS detector, found no discernible difference between the speed of a neutrino and the speed of light.[9] In May 2012, the Gran Sasso experiments BOREXINO, ICARUS, LVD and OPERA all measured neutrino velocity with a short-pulsed beam, and obtained agreement with the speed of light, showing that the original OPERA result was mistaken.[10] Finally in July 2012, the OPERA collaboration updated their results. After the instrumental effects mentioned above were taken into account, it was shown that the speed of 
neutrinos is consistent with the speed of light.[11] This was confirmed by a new, improved set of measurements in May 2013.[12]

https://www.wired.com/2012/06/neutrinos-cant-beat-light/

https://en.wikipedia.org/wiki/Measurements_of_neutrino_speed

\section{Copyrights}

Copyright for this article is retained by the author(s), with first publication rights granted to the journal.

This is an open-access article distributed under the terms and conditions of the Creative Commons Attribution license (http://creativecommons.org/licenses/by/4.0/). 